\title{
On the space-time monopole equation
}

\author{
Bo Dai, Chuu-Lian Terng, and Karen Uhlenbeck
}

\begin{abstract}
The space-time monopole equation is obtained from a dimension reduction of the anti-self dual Yang-Mills equation on $\mathbb{R}^{2,2}$. A family of Ward equations is obtained by gauge fixing from the monopole equation. In this paper, we give an introduction and a survey of the space-time monopole equation. Included are alternative explanations of results of Ward, Fokas-Ioannidou, Villarroel and Zakhorov-Mikhailov. The equations are formulated in terms of a number of equivalent Lax pairs; we make use of the natural Lorentz action on the Lax pairs and frames. A new Hamiltonian formulation for the Ward equations is introduced. We outline both scattering and inverse scattering theory and use Bäcklund transformations to construct a large class of monopoles which are global in time and have both continuous and discrete scattering data.
\end{abstract}

\section{Introduction}

The self-dual Yang-Mills equations in $\mathbb{R}^{4}$ and their reduction to monopole equations in $\mathbb{R}^{3}$ have become central topics of study and useful tools in modern geometry. The same self-dual equations in the case of a different signature of $\mathbb{R}^{2,2}$ are not of the general type to be used much in geometry. However, their dimensional reduction to the space-time monopole equation in $\mathbb{R}^{2,1}$ yields an extremely interesting system of non-linear wave equations which deserve to be better known. These equations can be encoded in a Lax pair. Moreover, with a mild additional assumption and a gauge fixing they can be rewritten for a map from $\mathbb{R}^{2,1}$ into a Lie group. These equations differ only slightly from the usual wave map equation.

This article is meant to be an introduction to and a survey of the literature on the space-time monopole equations. We also give a construction of the inverse scattering of the monopole equations via loop group factorizations. These equations form a hyperbolic system for a connection and a Higgs field, and hence have a gauge symmetry. A simple restriction and

The research of the second author was supported in part by NSF grant DMS-0529756. The research of the third author was supported in part by Sid Richardson Regents' Chair Funds, University of Texas system and NSF grant DMS-0305505. 
coordinate change produces the equation for a map into the gauge group. This last equation was introduced by Richard Ward, who studied them using a version of Riemann-Hilbert problem and twistor theory. He produced the basic examples and a number of interesting papers $[\mathbf{2 2}, \mathbf{2 3}, \mathbf{2 4}]$. Hence the equation for the map is referred to as either Ward's equation, or in his original language, the modified chiral model. Additional work on the equations is due to T. Ioannidou, W. Zakrewski $[\mathbf{1 0}, \mathbf{1 1}, \mathbf{1 2}]$, Manakov and Zakharov [13], A. K. Fokas and Ioannidou [7] and Villaroel [21]. The last three references present both the continuous scattering theory and the inverse scattering transform. The construction of a complete set of soliton solutions has been carried out by the first two authors in a previous paper $[6]$.

The plan of the paper is as follows. We derive the monopole equations with their Lax pairs, paying special attention to the difference between monopole equations in space and in space-time in Section 2. A family of Ward equations for maps into groups is constructed in Section 3. Section 4 describes the action of the Lorentz group on the Lax pair system and on frames. We make use of Lorentz boosts in the construction of solitons and of the spacial rotation group in deriving estimates in the appendix. Next we list special classes of solutions, so we can continue the discussion with a lot of examples in mind. Section 6 contains a very brief Hamiltonian formulation for the family of Ward equations. In Section 7, we introduce the transform which produces the continuous scattering data as well as the inverse scattering transform. Since the inverse scattering transform always exists, this produces many global solutions to the equations that are decaying at spacial infinity. The details of the fixed point theorem which yields continuous scattering data for small initial data are in the appendix. In Section 8 to 10, we review Bäcklund transformations, and use these transformations to construct soliton monopoles and monopoles with both continuous and discrete scattering data.

Due to soliton theory, Bäcklund transformations and the inverse scattering transform, we discover a very large class of solutions which are global in time. This is in contrast to the closely related wave map equation from $\mathbb{R}^{2,1}$ to $G$. It is a difficult theorem first of T. Tao $[\mathbf{1 5}]$, extended by D. Tataru [16], to show that small initial data results in solutions for all time. Whether the difference is entirely due to integrability, or whether there is a deeper analytic theory or more examples to be found, remains open.

\section{The Monopole equations}

The curvature $F_{A}=\sum_{i, j} F_{i j} d x_{i} \wedge d x_{j}$ of a $u(n)$-valued connection 1form $A=\sum_{i=1}^{4} A_{i} d x_{i}$ on $\mathbb{R}^{4}$ is

$$
F_{i j}=\left[\nabla_{i}, \nabla_{j}\right]=-\partial_{x_{i}}\left(A_{j}\right)+\partial_{x_{j}}\left(A_{i}\right)+\left[A_{i}, A_{j}\right],
$$

where

$$
\nabla_{i}=\partial_{x_{i}}-A_{i}
$$


The connection $A$ is anti-self-dual Yang-Mills (ASDYM) on $\mathbb{R}^{4}$ if

$$
* F_{A}=-F_{A},
$$

where $*$ is the Hodge star operator with respect to the Euclidean metric $\sum_{i=1}^{4} d x_{i}^{2}$ on $\mathbb{R}^{4}$. The ASDYM on $\mathbb{R}^{4}$ written in coordinates is

$$
F_{12}=-F_{34}, \quad F_{13}=-F_{42}, \quad F_{14}=-F_{23} .
$$

The ASDYM has a Lax pair formulation. The term "Lax pair" refers to any equation which is written as a "zero curvature" equation for a connection, or a portion of a connection. This connection contains an additional complex parameter $\mu$ which is variously interpreted as a "spectral", "twistor", or "Riemann-Hilbert parameter". Set

$$
z=x_{1}+i x_{2}, \quad w=x_{3}+i x_{4},
$$

$\nabla_{z}=\frac{1}{2}\left(\nabla_{1}-i \nabla_{2}\right)=\frac{\partial}{\partial z}-A_{z}, \nabla_{\bar{z}}=\frac{1}{2}\left(\nabla_{1}+i \nabla_{2}\right)=\frac{\partial}{\partial \bar{z}}-A_{\bar{z}}$, and $\nabla_{w}, \nabla_{\bar{w}}$ similarly. Since $A_{i} \in u(n), A_{\bar{z}}=-A_{z}^{*}$ and $A_{\bar{w}}=-A_{w}^{*}$, where $B^{*}=\bar{B}^{t}$. The equation

$$
\left[\nabla_{\bar{w}}+\mu \nabla_{z}, \nabla_{w}-\mu^{-1} \nabla_{\bar{z}}\right]=0
$$

is equivalent to the ASDYM (2.1) on $\mathbb{R}^{4}$. This is because (2.2) holds for all $\mu \in \mathbb{C} \backslash\{0\}$ if and only if the coefficients of $\mu, 1$ and $\mu^{-1}$ of (2.2) are zero, which is (2.1).

If we assume the ASDYM connection $A$ is independent of $x_{4}$, then $A_{w}=$ $\frac{1}{2}\left(A_{t}-i \Phi\right)$ and $A_{\bar{w}}=\frac{1}{2}\left(A_{t}+i \Phi\right)$, where $\Phi=A_{4}$ is the Higgs field, $A=$ $A_{t} d t+A_{z} d z+A_{\bar{z}} d \bar{z}$ is a connection 1 -form on $\mathbb{R}^{3}$. Then $(A, \Phi)$ satisfies the Euclidean monopole equation (cf. [3])

$$
D_{A} \Phi=* F_{A},
$$

where $*$ is the Hodge star operator with respect to the metric $d x^{2}+d y^{2}+$ $d t^{2}$. The Euclidean monopole equation is an important equation in both geometry and physics (cf. $[\mathbf{8}, \mathbf{3}]$ ).

The ASDYM on $\mathbb{R}^{2,2}$ is again $* F_{A}=-F_{A}$, but $*$ is the Hodge star operator with respect to the metric $d x_{1}^{2}+d x_{2}^{2}-d x_{3}^{2}-d x_{4}^{2}$. In coordinates, the ASDYM is

$$
F_{12}=F_{34}, \quad F_{13}=-F_{42}, \quad F_{14}=-F_{23} .
$$

This equation has a Lax pair similar to the ASDYM on $\mathbb{R}^{4}$,

$$
\left[\nabla_{\bar{w}}+\mu \nabla_{z}, \nabla_{w}+\mu^{-1} \nabla_{\bar{z}}\right]=0
$$

(the only difference with $(2.2)$ is that the second operator is taken with a plus sign). In other words, $A$ satisfies (2.3) if and only if (2.4) holds for all $\mu \in \mathbb{C} \backslash\{0\}$.

We say $E(\ldots, \mu)$ is a frame of the Lax pair $\left[D_{1}(\mu), D_{2}(\mu)\right]=0$ if $D_{1} E=$ $D_{2} E=0$.

If $\left[D_{1}(\mu), D_{2}(\mu)\right]=0$ is a Lax pair of a PDE with spectral parameter $\mu$, then $\left[\tilde{D}_{1}(\lambda), \tilde{D}_{2}(\lambda)\right]=0$ is also a Lax pair of the same PDE with parameter 
$\mu=\frac{a \lambda+b}{c \lambda+d}$, where $\tilde{D}_{i}(\lambda)=\sum_{j=1}^{2} f_{i j}(\mu) D_{j}(\mu)$ and $f_{i j}$ are meromorphic functions. Moreover, if $E$ is a frame for the Lax pair $\left[D_{1}(\mu), D_{2}(\mu)\right]=0$, then $F(\ldots, \lambda)=E\left(\ldots, \frac{a \lambda+b}{c \lambda+d}\right)$ is a frame for the Lax pair $\left[\tilde{D}_{1}(\lambda), \tilde{D}_{2}(\lambda)\right]=0$. We say these Lax pairs are equivalent.

If there is a $g$ such that $D_{1}\left(\mu_{0}\right) g=D_{2}\left(\mu_{0}\right) g=0$, then $g^{-1}\left[D_{1}(\mu)\right.$, $\left.D_{2}(\mu)\right] g=0$ is also a Lax pair for the same PDE, which is called the Lax pair obtained from the Lax pair $\left[D_{1}(\mu), D_{2}(\mu)\right]=0$ by fixing the gauge at $\mu=\mu_{0}$. So Lax pairs of a PDE come in many forms. We can choose an equivalent Lax pair and fix a gauge to make our computation easier or more transparent. For example, this is what we will do in later sections when we compute the $S O(2,1)$-actions on frames and discuss the scattering and inverse scattering of the monopole equations.

By making a linear fractional transformations in $\mu$, we obtain an equivalent Lax pair. Set $\mu=\frac{\lambda-i}{\lambda+i}$. Then

$$
\left\{\begin{array}{l}
2(\lambda+i)\left(\nabla_{\bar{w}}+\mu \nabla_{z}\right)=L_{1}+i L_{2} \\
2(\lambda-i)\left(\nabla_{w}+\mu^{-1} \nabla_{\bar{z}}\right)=L_{1}-i L_{2}
\end{array}\right.
$$

where

$$
\left\{\begin{array}{l}
L_{1}=\lambda\left(\nabla_{x_{3}}+\nabla_{x_{1}}\right)-\left(\nabla_{x_{4}}+\nabla_{x_{2}}\right), \\
L_{2}=\lambda\left(\nabla_{x_{4}}-\nabla_{x_{2}}\right)+\left(\nabla_{x_{3}}-\nabla_{x_{1}}\right) .
\end{array}\right.
$$

It follows that (2.4) holds if and only if $\left[L_{1}, L_{2}\right]=0$, i.e.,

$$
\left[\lambda\left(\nabla_{x_{3}}+\nabla_{x_{1}}\right)-\left(\nabla_{x_{4}}+\nabla_{x_{2}}\right), \lambda\left(\nabla_{x_{4}}-\nabla_{x_{2}}\right)+\left(\nabla_{x_{3}}-\nabla_{x_{1}}\right)\right]=0
$$

holds for all $\lambda \in \mathbb{C} \backslash\{i,-i\}$. So (2.6) is an equivalent Lax pair for the ASDYM on $\mathbb{R}^{2,2}$. The use of $\mu$ in the section on scattering theory, and $\lambda$ in the rest of the literature on the Ward equation, is unfortunately confusing, but necessary.

If we assume the connection $A$ is independent of $x_{4}$, then

$$
A_{w}=\frac{1}{2}\left(A_{t}-i \phi\right), \quad A_{\bar{w}}=\frac{1}{2}\left(A_{t}+i \phi\right)
$$

where $A_{x_{4}}=\phi$ is now the Higgs field for our new space-time monopole equation. A calculation shows that we can write the space-time monopole equation in the same form as the more familiar Euclidean monopole equation for a connection $A=A_{t} d t+A_{z} d z+A_{\bar{z}} d \bar{z}$ and a Higgs field $\phi$ (cf. [25]):

$$
D_{A} \phi=* F_{A} .
$$

Here we have used the $*$ operator for the Lorentz metric $d x^{2}+d y^{2}-d t^{2}$.

In this paper, we always assume $(A, \phi)$ decays at spacial infinity.

Equation (2.7) has a Lax pair induced from the Lax pair (2.4) for ASDYM:

$$
\left[\frac{1}{2} \nabla_{t}-\frac{i \phi}{2}+\mu \nabla_{z}, \quad \frac{1}{2} \nabla_{t}+\frac{i \phi}{2}+\mu^{-1} \nabla_{\bar{z}}\right]=0
$$


Set

$$
\mu=\frac{\lambda-i}{\lambda+i}, \quad \xi=\frac{t+x}{2}, \quad \eta=\frac{t-x}{2} .
$$

Then (2.6) induces an equivalent Lax pair for the monopole equation in $(\xi, \eta, y)$ coordinates:

$$
\left[\lambda \nabla_{\xi}-\nabla_{y}+\phi, \lambda^{-1} \nabla_{\eta}-\nabla_{y}-\phi\right]=0 .
$$

This is the Lax pair used by Ward. We have:

Proposition 2.1. The following statements are equivalent for a connection $A$ on $\mathbb{R}^{2,1}$ and a Higgs field $\phi$ :

(1) $(A, \phi)$ is a solution of the space time monopole equation (2.7) on $\mathbb{R}^{2,1}$.

(2) (2.8) holds for all $\mu \in \mathbb{C} \backslash\{0\}$.

(3) The linear system,

$$
\left\{\begin{array}{l}
\left(\frac{1}{2} \frac{\partial}{\partial t}+\mu \frac{\partial}{\partial z}\right) E=\left(\frac{1}{2}\left(A_{t}+i \phi\right)+\mu A_{z}\right) E \\
\left(\frac{1}{2} \frac{\partial}{\partial t}+\mu^{-1} \frac{\partial}{\partial \bar{z}}\right) E=\left(\frac{1}{2}\left(A_{t}-i \phi\right)+\mu^{-1} A_{\bar{z}}\right) E
\end{array}\right.
$$

is compatible for complex parameter $\mu$.

(4) (2.9) holds for all $\lambda \in \mathbb{C} \backslash\{0\}$.

(5) The linear system

$$
\left\{\begin{array}{l}
\left(\lambda \frac{\partial}{\partial \xi}-\frac{\partial}{\partial y}\right) F=\left(\lambda A_{\xi}-\left(A_{y}+\phi\right)\right) F \\
\left(\lambda^{-1} \frac{\partial}{\partial \eta}-\frac{\partial}{\partial y}\right) F=\left(\lambda^{-1} A_{\eta}-\left(A_{y}-\phi\right)\right) F
\end{array}\right.
$$

is compatible for complex parameter $\lambda$.

Moreover, if $E(x, y, t, \mu)$ is a frame of (2.8) (i.e., a solution of (2.10)), then

(a) $F(x, y, t, \lambda)=E\left(x, y, t, \frac{\lambda-i}{\lambda+i}\right)$ is a frame of (2.9) (i.e., a solution of $(2.11))$,

(b) E satisfies the reality condition

$$
E\left(x, y, t, \bar{\mu}^{-1}\right)^{*} E(x, y, t, \mu)=\mathrm{I}
$$

if and only if $F$ satisfies the reality condition

$$
F(x, y, t, \bar{\lambda})^{*} F(x, y, t, \lambda)=\mathrm{I} .
$$

\section{The Ward equations}

We call solutions of linear systems (2.10) or (2.11) that satisfy the reality condition monopole frames. Note that, unlike the case when the Lax pair is a full connection, locally there can be a serious lack of uniqueness in solving for a frame. We resolve this lack of uniqueness away from $\mu \in S^{1}$ by observing that the spacial part of the Lax pair is a Cauchy-Riemann operator. Frames, if they exist, are unique if we require $E_{\mu}=\mathrm{I}$ at spacial infinity. We expect the frames to exist at most points $\mu \notin S^{1}$. 
When $\mu=\rho \in S^{1}$, the existence of frames is more problematic. To obtain the Ward equation, we need extra assumptions, even for small initial data.

Definition 3.1. Let $\rho \in S^{1}$, and $(A, \phi)$ be a solution of the space-time monopole equation such that $(A, \phi)$ decays at spacial infinity. We say $(A, \phi)$ is $\rho$-regular, if there is a smooth solution $k: \mathbb{R}^{2,1} \rightarrow U(n)$ such that

$$
\left\{\begin{array}{l}
\left(\frac{1}{2} \partial_{t}+\rho \partial_{z}\right) k=\left(A_{\bar{w}}+\rho A_{z}\right) k \\
\left(\frac{1}{2} \partial_{t}+\rho^{-1} \partial_{\bar{z}}\right) k=\left(A_{w}+\rho^{-1} A_{\bar{z}}\right) k
\end{array}\right.
$$

and $k-\mathrm{I}$ and the first derivative of $k$ decays as $|z| \rightarrow \infty$. (Note that the second equation of (3.1) is the Hermitian transpose of the first.)

Let $f$ be a $U(n)$-valued map. Then

$$
f\left(\partial_{x}-A\right) f^{-1}=\partial_{x}-\left(f A f^{-1}+\left(\partial_{x} f\right) f^{-1}\right)
$$

is the gauge transformation of $f$ on $\frac{\partial}{\partial x}-A$, or

$$
f * A=f A f^{-1}+f_{x} f^{-1} .
$$

Suppose $(A, \phi)$ is $\rho$-regular and $k$ is the solution of $(3.1)$. We fix the gauge at $\mu=\rho$, i.e., we apply the gauge transformation of $k^{-1}$ to the Lax pair (2.8) to get

$$
\left[\frac{1}{2} \partial_{t}+\mu \partial_{z}-(\mu-\rho) \tilde{A}_{z}, \frac{1}{2} \partial_{t}+\mu^{-1} \partial_{\bar{z}}-\left(\mu^{-1}-\rho^{-1}\right) \tilde{A}_{\bar{z}}\right]=0,
$$

where $\tilde{A}_{z}=k^{-1} * A_{z}$ and $\tilde{A}_{\bar{z}}=k^{-1} * A_{\bar{z}}$. Or equivalently, the following linear system is compatible for an open subset of parameters $\mu$ :

$$
\left\{\begin{array}{l}
\left(\frac{1}{2} \frac{\partial}{\partial t}+\mu \frac{\partial}{\partial z}\right) E=(\mu-\rho) \tilde{A}_{z} E \\
\left(\frac{1}{2} \frac{\partial}{\partial t}+\mu^{-1} \frac{\partial}{\partial \bar{z}}\right) E=\left(\mu^{-1}-\rho^{-1}\right) \tilde{A}_{\bar{z}} E .
\end{array}\right.
$$

Suppose $(A, \phi)$ is also $-\rho$-regular. Then there exists $g: \mathbb{R}^{2,1} \rightarrow U(n)$ which satisfies (3.3) with $\mu=-\rho$, i.e.,

$$
\left\{\begin{array}{l}
\left(\frac{1}{2} \partial_{t}-\rho \partial_{z}\right) g=-2 \rho \tilde{A}_{z} g \\
\left(\frac{1}{2} \partial_{t}-\rho^{-1} \partial_{\bar{z}}\right) g=-2 \rho^{-1} \tilde{A}_{\bar{z}} g
\end{array}\right.
$$

A computation shows that

$(3.5)-\left(g_{t} g^{-1}\right)_{t}+\left(g_{x} g^{-1}\right)_{x}+\left(g_{y} g^{-1}\right)_{y}+\left[g_{t} g^{-1}, \cos \theta g_{x} g^{-1}+\sin \theta g_{y} g^{-1}\right]=0$, where $\rho=e^{i \theta}$. This is the one-parameter family of Ward equations [22]. We then obtain

Proposition 3.2. Suppose $E(x, y, t, \mu)$ is a frame for the solution of the space-time monopole equation, (i.e., $E$ is a solution of the linear system $(2.10))$, and $E(x, y, t, \mu)$ are smooth at $\mu= \pm e^{i \theta}$. Then

$$
g(x, y, t):=E\left(x, y, t, e^{i \theta}\right)^{-1} E\left(x, y, t,-e^{i \theta}\right)
$$

is a solution of the Ward equation (3.5). 
The Lax pair (2.9) is equivalent to the following Lax pair:

$$
\left[\lambda \nabla_{\xi}-\nabla_{y}+\phi, \lambda\left(\nabla_{y}+\phi\right)-\nabla_{\eta}\right]=0 .
$$

We fix the gauge of (3.6) at $\lambda=\infty$ (equivalent to fixing the gauge of (2.8) at $\mu=1$ ), i.e., take the gauge transformation of $h^{-1}$ on (3.6) to get

$$
\left[\lambda \partial_{\xi}-\left(\partial_{y}-\hat{A}\right), \quad \lambda \partial_{y}-\left(\frac{\partial}{\partial \eta}-\hat{B}\right)\right]=0,
$$

where $\partial_{y} h=\left(A_{y}-\phi\right) h, \partial_{\xi} h=A_{\xi} h, \hat{A}=-2 h^{-1} \phi h$ and $\hat{B}=h^{-1} * A_{\eta}$. So we have the following proposition:

Proposition 3.3. The following statements are equivalent:

(1) Equation (3.7) holds for all $\lambda \in \mathbb{C}$,

(3) The linear system

$$
\left\{\begin{array}{l}
\partial_{\xi} \hat{B}=\partial_{y} \hat{A}, \\
{\left[\partial_{y}-\hat{A}, \partial_{\eta}-\hat{B}\right]=0 .}
\end{array}\right.
$$

$$
\left\{\begin{array}{l}
\left(\lambda \partial_{\xi}-\partial_{y}\right) H=-\hat{A} H \\
\left(\lambda \partial_{y}-\partial_{\eta}\right) H=-\hat{B} H
\end{array}\right.
$$

is locally solvable for an open subset of $\lambda \in \mathbb{C}$. Moreover, if $H(x, y, t, \lambda)$ is a solution of (3.8) and is smooth at $\lambda=0$, then $g=H(\ldots, 0)$ satisfies

$$
\frac{\partial}{\partial t}\left(\frac{\partial g}{\partial t} g^{-1}\right)-\frac{\partial}{\partial x}\left(\frac{\partial g}{\partial x} g^{-1}\right)-\frac{\partial}{\partial y}\left(\frac{\partial g}{\partial y} g^{-1}\right)-\left[\frac{\partial g}{\partial t} g^{-1}, \frac{\partial g}{\partial x} g^{-1}\right]=0
$$

i.e., $g$ is a solution of Ward equation (3.5) with $\theta=0$.

As a consequence of the above proposition, we see that to construct solutions of the monopole equation that are $\lambda=\infty$-regular, it suffices to construct $H(\xi, \eta, y, \lambda)$ such that $\left(\lambda \partial_{\xi} H-\partial_{y} H\right) H^{-1}$ and $\left(\lambda \partial_{y} H-\partial_{\eta} H\right) H^{-1}$ are independent of $\lambda$.

Proposition 3.4. If a monopole is $\mu= \pm 1$ regular (i.e., $\lambda=\infty, 0$ regular $)$, then it is gauge equivalent to a monopole $(A, \phi)$ such that $A_{\xi}=0$, $A_{y}=\phi$. Conversely, if $(\hat{A}, \hat{B})$ satisfies (3.7), then $A_{\xi}=0, A_{y}=\phi=\hat{A} / 2$, and $A_{\eta}=\hat{B}$ is a monopole.

\section{The Action of $S O(2,1)$}

The Lorentz group $S O(2,1)$ is the group of all $g \in S L(3, \mathbb{R})$ such that $g^{t} \mathrm{I}_{2,1} g=\mathrm{I}_{2,1}$, where $\mathrm{I}_{2,1}=\operatorname{diag}(1,1,-1)$. The group $S O(2,1)$ acts on $\mathbb{R}^{2,1}$ by the standard action $g \cdot p=g p$ (here $p \in \mathbb{R}^{2,1}$ is identified as a $3 \times 1$ vector). Given a connection $A=A_{1} d x+A_{2} d y+A_{3} d t$, a Higgs field $\phi$, and $g \in S O(2,1)$, the action $g \cdot(A, \phi)=(g \cdot A, g \cdot \phi)$ is defined by $g \cdot A=g \cdot A_{1} d x+g \cdot A_{2} d y+g \cdot A_{3} d t$, where

$$
\left(g \cdot A_{i}\right)(p)=A_{i}(g \cdot p), \quad(g \cdot \phi)(p)=\phi(g \cdot p) .
$$


The space-time monopole equation is invariant under the Lorentz group $S O(2,1)$, i.e., if $(A, \phi)$ is a solution then so is $g \cdot(A, \phi)=(g \cdot A, g \cdot \phi)$ for $g \in S O(2,1)$. In order to make the scattering theory estimates tractable and to understand the 1-solitons we need to understand the natural action of $S O(2,1)$ on frames (solutions of linear system (2.11)). Since $S O(2)$ of the $x y$-plane and $O(1,1)$ of the $x t$-plane generate $S O(2,1)$, to compute the explicit formula of the action of $S O(2,1)$ on frames, it suffices to compute the action of the following one-parameter subgroups on frames:

$$
\begin{aligned}
& R(\theta)=\left(\begin{array}{ccc}
\cos \theta & -\sin \theta & 0 \\
\sin \theta & \cos \theta & 0 \\
0 & 0 & 1
\end{array}\right) \\
& T(s)=\left(\begin{array}{ccc}
\cosh s & 0 & \sinh s \\
0 & 1 & 0 \\
\sinh s & 0 & \cosh s
\end{array}\right)
\end{aligned}
$$

We also need the representation $\sigma: S O(2,1) \rightarrow S L(2, \mathbb{R})$, whose differential $d \sigma_{e}$ maps $e_{12}-e_{21}$ to $-\frac{1}{2}\left(e_{12}-e_{21}\right), e_{13}+e_{31}$ to $\frac{1}{2}\left(e_{11}-e_{22}\right)$, and $e_{23}+e_{32}$ to $-\frac{1}{2}\left(e_{12}+e_{21}\right)$. So

$$
\sigma(R(\theta))=\left(\begin{array}{cc}
\cos \frac{\theta}{2} & \sin \frac{\theta}{2} \\
-\sin \frac{\theta}{2} & \cos \frac{\theta}{2}
\end{array}\right), \quad \sigma(T(s))=\left(\begin{array}{cc}
e^{\frac{s}{2}} & 0 \\
0 & e^{-\frac{s}{2}}
\end{array}\right) .
$$

The group $S L(2, \mathbb{R})$ acts on the scattering parameter space $\mathbb{C} \cup\{\infty\}$ by the linear fractional transformations:

$$
\left(\begin{array}{ll}
a & b \\
c & d
\end{array}\right) * \lambda=\frac{a \lambda+b}{c \lambda+d}
$$

THEOREM 4.1. The group $S O(2,1)$ acts on the Lax pairs and on the frames of the space-time monopole equation. If $F$ is a frame of $(A, \phi)$, then

$$
(g \cdot F)(p, \lambda)=F(g \cdot p, \sigma(g) * \lambda)
$$

is a frame for $g \cdot(A, \phi)$, where $\sigma: S O(2,1) \rightarrow S L(2, \mathbb{R})$ is the representation given above and $*$ is the standard action of $S L(2, \mathbb{R})$ on $\lambda$ via the linear fractional transformation.

Proof. For the action of $R(\theta)$, it can be checked easily that if $E$ is a solution of (2.10) for $(A, \phi)$, then $\tilde{E}$ solves $(2.10)$ for $e^{i \theta} \cdot(A, \Phi)$, where

$$
\tilde{E}(z, t, \mu)=E\left(e^{i \theta} z, t, e^{i \theta} \mu\right)
$$

(Here we identify $e^{i \theta}$ with the rotation matrix $R(\theta)$ ). In other words,

$$
R(\theta) \cdot E(p, \mu)=E\left(R(\theta) p, e^{i \theta} \mu\right)
$$

To compute the action of $R(\theta)$ on solutions $F$ of $(2.11)$, we recall that $\mu=\frac{\lambda-i}{\lambda+i}, \lambda=\frac{i(1+\mu)}{1-\mu}$, and

$$
F(p, \lambda)=E\left(p, \frac{\lambda-i}{\lambda+i}\right)
$$


where $E$ is a solution of (2.10). But for $\tilde{\mu}=e^{i \theta} \mu$, the corresponding $\tilde{\lambda}$ and $\lambda$ are related by

$$
\tilde{\lambda}=\frac{i\left(1+e^{i \theta}\right) \lambda-\left(1-e^{i \theta}\right)}{\left(1-e^{i \theta}\right) \lambda+i\left(1+e^{i \theta}\right)}=\frac{\cos \frac{\theta}{2} \lambda+\sin \frac{\theta}{2}}{-\sin \frac{\theta}{2} \lambda+\cos \frac{\theta}{2}}=\left(\begin{array}{cc}
\cos \frac{\theta}{2} & \sin \frac{\theta}{2} \\
-\sin \frac{\theta}{2} & \cos \frac{\theta}{2}
\end{array}\right) * \lambda .
$$

In other words,

$$
(R(\theta) \cdot F)(p, \lambda)=F(R(\theta) p, \sigma(R(\theta)) * \lambda) .
$$

For the action of $T(s)$, we use the light cone coordinates $\xi, \eta$. Then $T(s)$ maps $(\xi, \eta, y)$ to $\left(e^{s} \xi, e^{-s} \eta, y\right)$. Suppose $F(p, \lambda)$ is a solution of linear system (2.11) for $(A, \phi)$. Given $s \in \mathbb{R}$, define

$$
\hat{F}(\xi, \eta, y, \lambda)=F\left(T(s) \cdot p, e^{s} \lambda\right)=F\left(e^{s} \xi, e^{-s} \eta, y, e^{s} \lambda\right)
$$

It can be checked easily that $\hat{F}$ is a frame for $T(s) \cdot(A, \phi)$. But

$$
e^{s} \lambda=\left(\begin{array}{cc}
e^{\frac{s}{2}} & 0 \\
0 & e^{-\frac{s}{2}}
\end{array}\right) * \lambda=\sigma(T(s)) * \lambda,
$$

where $*$ is the standard action of $S L(2, \mathbb{R})$ on $\mathbb{C}$.

Corollary 4.2. The group $S O(2,1)$ acts on frames $E$ of $(2.10)$ as follows: If $E$ is a frame of $(A, \Phi)$, then $(g \cdot E)(p, \mu)=E(g \cdot p, g \sharp \mu)$, where

$$
\begin{aligned}
R(\theta) \sharp \mu & =e^{i \theta} \mu, \\
T(s) \sharp \mu & =\frac{\cosh \frac{s}{2} \mu+\sinh \frac{s}{2}}{\sinh \frac{s}{2} \mu+\cosh \frac{s}{2}} .
\end{aligned}
$$

\section{Special classes of solutions}

Many examples of solutions to the monopole equations are obtained by assuming additional conditions. A first set of examples comes from linear solutions of the wave equation. If $H \subset G$ is any abelian subgroup, the monopole equations for maps into $H$ are linear. This provides us with a number of solutions to which we can later apply Bäcklund transformations. Let $\mathcal{H} \subset \mathcal{G}$ be the Lie subalgebra of $H$. Suppose that $(A, \phi)=\left(a, a_{0}, \phi\right) \in$ $\oplus^{2} \mathfrak{h} \oplus \mathfrak{h} \oplus \mathfrak{h}$ decays at spacial infinity, where $a_{0}=A_{t}, a=\left(a_{1}, a_{2}\right)=\left(A_{x}, A_{y}\right)$, and $\mathfrak{h}$ is the Lie algebra of $H$. Then the monopole equations (2.7) are written in space-time (here $d$ and $*$ are spacial):

$$
\left\{\begin{array}{l}
* d a=\frac{\partial \phi}{\partial t} \\
\frac{\partial a}{\partial t}-d a_{0}=* d \phi
\end{array}\right.
$$

The gauge transformation of $e^{-u}$ appears as

$$
\left(a, a_{0}, \phi\right) \mapsto\left(a-d u, a_{0}-\frac{\partial u}{\partial t}, \phi\right) .
$$


A global way of fixing gauge suitably is to require that $d * a=0$ (a spacial equation which is easily solved). With this choice, we apply $d *$ to the second equation of (5.1) to obtain

$$
\frac{\partial}{\partial t}(d * a)-d * d a_{0}=d * * d \phi=0
$$

Since $d * a=0, \triangle a_{0}=0$. We assume $a_{0}$ decays at infinity, hence $a_{0}=0$. Finally, we conclude

Proposition 5.1. The abelian monopole equations are equivalent to the linear wave equation for $\phi$ :

$$
\square \phi=\left(\partial_{t}^{2}-\partial_{x}^{2}-\partial_{y}^{2}\right) \phi=0,
$$

with $* d a=\frac{\partial \phi}{\partial t}, d * a=0$, and $a_{0}=0$.

We make special note of the fact that the condition of $\rho$-regular is not automatic even in the abelian case. It is satisfied if we can find a gauge transformation $g=e^{u} \in H$ such that for $\rho=e^{i \alpha}$ we have $D_{1}(\rho) g=D_{2}(\rho) g=0$. But

$$
D_{1}(\rho)-D_{2}(\rho)=i\left(\sin \alpha \nabla_{x}-\cos \alpha \nabla_{y}-\phi\right)
$$

so we have $g^{-1}\left(D_{1}(\rho)-D_{2}(\rho)\right) g=i\left(\sin \alpha \frac{\partial}{\partial x}-\cos \alpha \frac{\partial}{\partial y}\right)$, which implies that

$$
-\sin \alpha\left(a_{1}-\frac{\partial u}{\partial x}\right)+\cos \alpha\left(a_{2}-\frac{\partial u}{\partial y}\right)-\phi=0 .
$$

Here $u$ decays at spacial infinity. But (5.2) is the ODE

$$
\frac{d u}{d \tau}=a_{1} \sin \alpha-a_{2} \cos \alpha+\phi,
$$

where $d \tau=\sin \alpha d x-\cos \alpha d y$. The condition that $u$ decays at spacial infinity is given by integral conditions, so it is not automatically true. However, if we can find a decaying solution of (5.2), it is unique. Because all the variables satisfy wave equations, if we find a solution $u$ for (5.2) at time $t=0$, we can propagate it using the wave equation to any $t$. It follows that the condition of $\rho$-regular is a property of the initial data alone.

Our second class of examples is well-known (cf. [20]). It involves solutions of the monopole equation which are invariant under time translation.

Proposition 5.2. Suppose that $(A, \phi)$ is a solution of the monopole equation, which is fixed under time translation. Then its Lax pair is gauge equivalent to the Lax pair of a harmonic map.

Proof. The Lax pair (2.8) becomes

$$
\left[\mu \nabla_{z}-\frac{1}{2}\left(A_{t}+i \phi\right), \mu^{-1} \nabla_{\bar{z}}-\frac{1}{2}\left(A_{t}-i \phi\right)\right]=0 .
$$

Since the connection at $\mu=1$ is now a full connection in $\mathbb{C}$, we fix the gauge at $\mu=1$, so the Lax pair is now a Lax pair for the harmonic map

$$
\left[\frac{\partial}{\partial z}+\frac{1-\mu^{-1}}{2} g^{-1}\left(A_{t}+i \phi\right) g, \frac{\partial}{\partial \bar{z}}+\frac{1-\mu}{2} g^{-1}\left(A_{t}-i \phi\right) g\right]=0
$$


where $g: \mathbb{C} \rightarrow U(n)$ satisfies

$$
\left\{\begin{array}{l}
\frac{\partial g}{\partial z} g^{-1}=A_{z}+\frac{1}{2}\left(A_{t}+i \phi\right), \\
\frac{\partial g}{\partial \bar{z}} g^{-1}=A_{\bar{z}}+\frac{1}{2}\left(A_{t}-i \phi\right) .
\end{array}\right.
$$

Note that this differs from the Euclidean monopole reduction, where the different sign produces Hitchin's self-dual equation ([9]) rather than the harmonic map.

COROLlary 5.3. The Lorentz transformations of the stationary solution corresponding to harmonic maps produce families of solutions to the monopole equations.

It can be checked that the solutions obtained in the above corollary decay in space.

Special solutions of the monopole equations also come from the reduction to $\mathbb{R}^{1,1}$, which involves the assumption that the field $(A, \phi)$ is independent of one of the spacial variables, say $y$. The solution of these equations will not directly yield solutions of the monopole equations which decay in space, but we will see that they do arise in the consideration of radially symmetric solutions.

Assume $(A, \phi)$ is independent of $y$. Then the $A_{y}=\psi$ becomes a second Higgs field and the Lax pair (2.8) reduces to

$$
\left[\nabla_{t}-i \phi+\mu\left(\nabla_{x}+i \psi\right), \nabla_{t}+i \phi+\mu^{-1}\left(\nabla_{x}-i \psi\right)\right]=0 .
$$

The equations become

$$
\left\{\begin{array}{l}
{\left[\nabla_{t}, \nabla_{x}\right]=[\phi, \psi],} \\
\nabla_{t} \psi=\nabla_{x} \phi, \\
\nabla_{t} \phi=\nabla_{x} \psi .
\end{array}\right.
$$

The usual Lax pair is obtained by restriction to characteristic coordinates. Let $\nabla_{t}+\nabla_{x}=\nabla_{\xi}, \nabla_{t}-\nabla_{x}=\nabla_{\eta}$, and $\phi_{+}=\phi+\psi, \phi_{-}=\psi-\phi$. We get three equations of $\phi_{+}$and $\phi_{-}$encoded in the Lax pair

$$
\left[\nabla_{\xi}+\tau \phi_{+}, \nabla_{\eta}+\tau^{-1} \phi_{-}\right]=0 .
$$

The wave map $\phi: \mathbb{R}^{1,1} \rightarrow G$ is obtained from this Lax pair in the same manner that the Ward map is obtained from the Lax pair for monopoles.

Finally we are interested in solutions with a radial symmetry. In gauge theory, the symmetry is inspired by requiring that under the pull-back of a space-time symmetry, the fields $(A, \phi)$ go to gauge transformations of themselves. For example, given $J \in s u(n)$ such that $e^{2 \pi J}=\mathrm{I}$, then

$$
e^{i \theta} \cdot(A, \phi)(z, t)=\left(e^{J \theta} A\left(e^{i \theta} z, t\right) e^{-J \theta}, e^{J \theta} \phi\left(e^{i \theta} z, t\right) e^{-J \theta}\right)
$$

is an $S O(2)$-action. If the monopole equation is invariant under this action, then the field

$$
(A, \phi)=\left(e^{J \theta} a e^{-J \theta}, e^{J \theta} a_{0} e^{-J \theta}, e^{J \theta} \phi e^{-J \theta}\right),
$$


where $a, a_{0}$ and $\phi$ depend only on $r$ and $t$. Both $a$ and $\phi$ must vanish at $r=0$ unless $J=0$. We then make a singular gauge transformation by $e^{J \theta}$ to the form

$$
(A, \phi)=\left(a_{r}, a_{\theta}-J, a_{0}, \phi\right),
$$

where $J$ now indicates a singularity at 0 and lack of suitable decay at $\infty$.

Proposition 5.4. Fix a representation of $S^{1} \rightarrow U(n)$ given by $e^{i \theta} \mapsto$ $e^{J \theta}$. The monopole equations for a monopole with radial symmetry induced by $J$ are equivalent to the equations for $\nabla_{r}, \nabla_{t}, \phi$ and $\psi=\frac{a_{\theta}-J}{r}$ :

$$
\left\{\begin{array}{l}
{\left[\nabla_{t}, \nabla_{r}\right]=[\phi, \psi],} \\
{\left[\nabla_{t}, \psi\right]=\left[\nabla_{r}, \phi\right],} \\
{\left[\nabla_{t}, \phi\right]=\left[\nabla_{r}, \psi\right]+\frac{1}{r} \psi .}
\end{array}\right.
$$

Proof. It is useful to notice that

$$
\begin{aligned}
& \nabla_{z}=\frac{1}{2}\left(\nabla_{x}-i \nabla_{y}\right)=\frac{e^{-i \theta}}{2}\left(\nabla_{r}-\frac{i}{r} \nabla_{\theta}\right), \\
& \nabla_{\bar{z}}=\frac{1}{2}\left(\nabla_{x}+i \nabla_{y}\right)=\frac{e^{i \theta}}{2}\left(\nabla_{r}+\frac{i}{r} \nabla_{\theta}\right) .
\end{aligned}
$$

Substitute these expression in the Lax pair (2.8) and compare coefficients of $\mu, \mu^{-1}$ and the constant term to get

$$
\left[\nabla_{t}-i \phi, \nabla_{r}+i \psi\right]=0,
$$

which yields two equations. The third equation come from the equation

$$
\left[\nabla_{t}-i \phi, \nabla_{t}+i \phi\right]+\left[e^{-i \theta}\left(\nabla_{r}-\frac{i}{r} \nabla_{\theta}\right), e^{i \theta}\left(\nabla_{r}+\frac{i}{r} \nabla_{\theta}\right)\right]=0 .
$$

This last equation has an extra term which spoils the integrability of the system, and prevents the three equations from being encoded as a Lax pair.

\section{Hamiltonian structures}

Let $(p, q)$ denote the standard variables for the cotangent bundle $\mathcal{M}$ of the space of rapidly decaying maps from $\mathbb{R}^{2}$ to $U(n)$, and $H: \mathcal{M} \rightarrow \mathbb{R}$ the functional defined by

$$
H=\frac{1}{2} \iint_{\mathbb{R}^{2}}\|p\|^{2}+\left\|q^{-1} d q\right\|^{2} d x d y .
$$

We introduce a Hamiltonian formulation of the Ward equation, which does not seem to have appeared in the literature before. For each unit direction $v$ of $\mathbb{R}^{2}$, (i) the symplectic form $w^{v}$ is the sum of the standard symplectic form on cotangent bundle $\mathcal{M}$ and an extra closed 2-form depending on $v$, (ii) the Hamiltonian system of $H$ with respect to $w^{v}$ is the Ward equation with $\rho=v$.

Let $\Theta$ be the canonical 1-form on $\mathcal{M}$ defined by

$$
\Theta_{(p, q)}\left(\delta p, q^{-1} \delta q\right)=p\left(q^{-1} \delta q\right) .
$$


The standard symplectic form on $\mathcal{M}$ is $d \Theta$. We identify the tangent and cotangent spaces in the Lie algebra formulation with the $L^{2}$ inner product

$$
\langle A, B\rangle=\int_{\mathbb{R}^{2}} \operatorname{tr}(A B) d x d y .
$$

Use the Cartan formula

$$
d \Theta\left(\xi_{1}, \xi_{2}\right)=\xi_{1}\left(\Theta\left(\xi_{2}\right)\right)-\xi_{2}\left(\Theta\left(\xi_{1}\right)\right)-\Theta\left(\left[\xi_{1}, \xi_{2}\right]\right)
$$

to compute $d \Theta$ to get

$$
\begin{aligned}
& d \Theta_{(p, q)}\left(\left(\delta_{1} p, q^{-1} \delta_{1} q\right),\left(\delta_{2} p, q^{-1} \delta_{2} q\right)\right) \\
& =\left\langle\delta_{1} p, q^{-1} \delta_{2} q\right\rangle-\left\langle\delta_{2} p, q^{-1} \delta_{1} q\right\rangle-\left\langle p,\left[q^{-1} \delta_{1} q, q^{-1} \delta_{2} q\right]\right\rangle .
\end{aligned}
$$

A computation shows that the following 2 -form is closed:

$$
\tau_{(p, q)}^{v}\left(\left(\delta_{1} p, q^{-1} \delta_{1} q\right),\left(\delta_{2} p, q^{-1} \delta_{2} q\right)\right)=\left\langle q^{-1} q_{v},\left[q^{-1} \delta_{1} q, q^{-1} \delta_{2} q\right]\right\rangle,
$$

where $q_{v}=d q(v)=q_{x} \cos \theta+q_{y} \sin \theta$ if $v=e^{i \theta}$. Then $w^{v}=d \Theta+\tau^{v}$ is a symplectic form on $\mathcal{M}$. In fact,

$$
\begin{aligned}
& w_{(p, q)}^{v}\left(\left(\delta_{1} p, q^{-1} \delta_{1} q\right),\left(\delta_{2} p, q^{-1} \delta_{2} q\right)\right) \\
& =\left\langle\delta_{1} p, q^{-1} \delta_{2} q\right\rangle-\left\langle\delta_{2} p, q^{-1} \delta_{1} q\right\rangle+\left\langle-p+q^{-1} q_{v},\left[q^{-1} \delta_{1} q, q^{-1} \delta_{2} q\right]\right\rangle .
\end{aligned}
$$

Since

$$
d H_{(p, q)}(\delta p, \delta q)=\langle\delta p, p\rangle-\left\langle q^{-1} \delta q, d^{*}\left(q^{-1} d q\right)\right\rangle,
$$

a direct computation shows that the Hamiltonian flow for $H$ with respect to the symplectic structure $w^{v}$ is

$$
\left\{\begin{array}{l}
q^{-1} q_{t}=p, \\
p_{t}+\left[-p+q^{-1} q_{v}, q^{-1} q_{t}\right]=d^{*}\left(q^{-1} d q\right) .
\end{array}\right.
$$

So we have proved

Proposition 6.1. Given a unit vector $v=e^{i \theta}$ in $\mathbb{R}^{2}$, the 2 -form $w^{v}$ is a symplectic form, and the Hamiltonian system of $H$ with respect to $w^{v}$ is the Ward equation (3.5).

In [12], Ioannidou and Zakrzewski considered another family of Ward equations, and studied the Lagrangian and Hamiltonian formulations for that family of equations.

\section{Scattering theory}

Scattering theory for the Ward equations has been treated by a number of authors, including Manakov and Zakharov [13], Villarroel [21], and Fokas and Ioannidou [7]. We include a brief synopsis and interpretation of the results. In particular, we construct the inverse scattering transform via loop group factorizations. 
Ward's original analysis of the space-time monopole equations is via twistor theory. We recognize features of this analysis in what follows, although we do not go into the twistor formulation. Recall that

$$
D_{1}(\mu)=\frac{1}{2} \nabla_{t}-\frac{i \phi}{2}+\mu \nabla_{z}, \quad D_{2}(\mu)=\frac{1}{2} \nabla_{t}+\frac{i \phi}{2}+\mu^{-1} \nabla_{\bar{z}} .
$$

We rewrite the Lax pair as the linear system consisting of first a spacial operator

$$
\begin{aligned}
D_{s}(\mu) & =D_{1}(\mu)-D_{2}(\mu)=\mu \nabla_{z}-\mu^{-1} \nabla_{\bar{z}}-i \phi \\
& =\frac{\left(\mu-\mu^{-1}\right)}{2} \nabla_{x}-i \frac{\left(\mu+\mu^{-1}\right)}{2} \nabla_{y}-i \phi .
\end{aligned}
$$

The second operator in the Lax pair we write as one of a family of time operators

$$
\begin{aligned}
D_{t}(\mu) & =D_{1}(\mu)+D_{2}(\mu)+\alpha D_{s}(\mu) \\
& =\nabla_{t}+\frac{1}{2}\left(\mu+\mu^{-1}\right) \nabla_{x}-\frac{i}{2}\left(\mu-\mu^{-1}\right) \nabla_{y}+\alpha D_{s}(\mu) .
\end{aligned}
$$

The operators can be rescaled (i.e., multiplied by a scalar function of $\mu$ ), so the points $\mu=0$ and $\mu=\infty$ are included by changing the scaling factor (for example, if we multiply by $\mu$ to the operators, then they are defined at $\mu=0$, and if we multiply by $\mu^{-1}$ to the operators, then they are defined at $\mu=\infty)$. Note that $D_{1} E=D_{2} E=0$ if and only if $D_{s} E=D_{t} E=0$.

If $\mu \notin S^{1}$, then the spacial part of the connection, $D_{s}(\mu)$, can be thought of as containing a $\bar{\partial}$ operator in the complex structure on $\mathbb{R}^{2}$ given by the complex coordinate

$$
w=\frac{\mu^{-1} z+\mu \bar{z}}{a(\mu)}, \quad \bar{w}=\frac{\bar{\mu}^{-1} \bar{z}+\bar{\mu} z}{\bar{a}(\mu)} .
$$

(In fact, $\left.D_{s}(\mu)=\nabla_{\bar{w}}\right)$. The factor $a(\mu)$ does not change the complex structure. Note at $\mu=\infty, w=\bar{z}$, and at $\mu=0, w=z$.

Suppose the Higgs field $\phi$ and the connection $A$ decay at infinity. For fixed time and every $\mu \in \mathbb{C} \cup\{\infty\} \backslash S^{1}$ the connection $D_{s}(\mu)$ determines a bundle holomorphic in the complex parameter $w=w(\mu)$ on $\mathbb{R}^{2} \cup\{\infty\}=S^{2}$. By a theorem of Grothendieck, this bundle is the sum of line bundles

$$
L_{\mu}=L_{1} \oplus L_{2} \oplus \cdots \oplus L_{n}
$$

with first Chern classes $c(1) \leq c(2) \leq \cdots \leq c(n)$. The reality condition insures that the Chern classes at $\bar{\mu}^{-1}$ are the negative of the Chern classes at $\mu$, since the bundle $L_{\bar{\mu}^{-1}}$ is dual to the bundle $L_{\mu}$ at $\mu$. We call this sequence $\vec{c}(\mu)=(c(1), \ldots, c(n))$ the Chern vector at $\mu$.

THEOREM 7.1. Suppose we have a solution of the monopole equation in a time interval $\left[T_{1}, T_{2}\right]$. Then for every $\mu \in \mathbb{C} \cup\{\infty\} \backslash S^{1}$, the Chern vector of the spacial holomorphic bundle is preserved under the flow in time. 
Proof. As a warm-up, we first prove this for $\mu=0$. Then the spacial connection is $\nabla_{\bar{z}}$ and we choose $D_{1}(0)=\nabla_{t}-i \phi$ as the evolution operator. We have $\left[\nabla_{t}-i \phi, \nabla_{\bar{z}}\right]=0$. We may make a complex gauge transformation so that $D_{1}(0)=\nabla_{t}-i \phi=h^{-1} \circ \frac{\partial}{\partial t} \circ h$. Hence the gauge equivalent Lax pair gives

$$
\frac{\partial}{\partial t}\left(h \circ \nabla_{\bar{z}} \circ h^{-1}\right)=0 .
$$

The complex structure on the bundle determined by $\nabla_{\bar{z}}$ is carried into a structure which is a gauge equivalent one. Hence they have the same splitting, and so the same Chern vectors.

A similar computation occurs for each $\mu$, where we make the choice of the time direction at $\mu$ to be real. For simplicity, let $\mu=e^{i \theta} \rho$ where $\rho \in \mathbb{R}^{+}$ and rotate variable by $z \mapsto e^{i \theta} z$ so that in this coordinate system $\mu=\rho$ is real. Choose as a suitable evolution operator

$$
D_{1}(\rho)+\rho^{2} D_{2}(\rho)=\frac{1+\rho^{2}}{2} \nabla_{t}+\rho \nabla_{x}-\frac{i\left(1-\rho^{2}\right) \phi}{2} .
$$

The derivatives which appear are in the direction of $\frac{\partial}{\partial \tau}=\frac{1+\rho^{2}}{2} \frac{\partial}{\partial t}+\rho \frac{\partial}{\partial x}$. We can again make a gauge transformation so that

$$
\begin{aligned}
& \nabla_{\tau}-\frac{1-\rho^{2}}{2} i \phi=h^{-1} \circ \frac{\partial}{\partial \tau} \circ h, \\
& \frac{\partial}{\partial \tau}\left(h \circ D_{s}(\rho) \circ h^{-1}\right)=0 .
\end{aligned}
$$

Hence the complex structure of the bundle does not changes under a flow in the $\tau$ variable. However, time translation is a translation in the $\tau$ direction followed by a translation in the $x$ variable. Translation in $S^{2}$ by $x$ is holomorphic and does not change the complex structure. Hence the splitting type of $L_{\mu}=L_{\rho}$ does not change under the flow in time. We conclude that the Chern vector $\vec{c}(\mu)$ is preserved.

What do we expect? Since the space of $\bar{\partial}$ derivatives which lead to non-trivial splitting has codimension at least two, we expect that for most choices of initial data, the non-trivial splitting occurs at isolated points. At these points in $\mathbb{C} \backslash S^{1}$, we expect to have singularities (poles and zeros). We expect continuous scattering data to be defined as a jump across $\mu \in S^{1}$.

Definition 7.2. A rapidly decaying spacial pair $(A, \phi)$ is said to have continuous scattering data if the frame, which solves

$$
\left\{\begin{array}{l}
D_{s}(\mu) E_{\mu}=\left(\frac{\mu-\mu^{-1}}{2} \nabla_{x}-\frac{i\left(\mu+\mu^{-1}\right)}{2} \nabla_{y}-i \phi\right) E_{\mu}=0 \\
E_{\mu}(\infty)=\mathrm{I}, \quad E_{\bar{u}^{-1}}=\left(E_{\mu}^{*}\right)^{-1}
\end{array}\right.
$$

has solutions $E_{\mu}^{ \pm}$, which are holomorphic in a

$$
\mathcal{O}_{\epsilon}^{ \pm}=\left\{\left.\mu \in \mathbb{C}|1<| \mu\right|^{ \pm 1}<1+\epsilon\right\}
$$


for some $\epsilon>0$. Moreover, we assume that the limits

$$
\lim _{\mu \in \mathcal{O}^{ \pm}, \mu \rightarrow e^{i \theta}} E_{\mu}=S_{\theta}^{ \pm}
$$

exist. It follows from the reality condition that $S_{\theta}^{-}=\left(S_{\theta}^{+}\right)^{*-1}$. We call the non-negative Hermitian matrix

$$
S_{\theta}=\left(S_{\theta}^{-}\right)^{-1} S_{\theta}^{+}=\left(S_{\theta}^{+}\right)^{*} S_{\theta}^{+}
$$

the scattering matrix.

Let $W^{2,1}$ denote the space of maps $f$ such that $f$ and first partial derivatives of $f$ are in $L^{1}$.

Proposition 7.3. Assume there is a gauge in which $(A, \phi)$ is rapidly decaying in spacial variables; moreover, assume $(A, \phi)$ is small in $W^{2,1}$. Then the Chern vector $\vec{c}(\mu)=0$ at every $\mu \in \mathbb{C} \cup\{\infty\} \backslash S^{1}$. Moreover, the continuous scattering matrix $S_{\theta}$ exists, I- $S_{\theta}$ decays for each $\theta$, and the scattering matrix $S_{\theta}$ satisfies

(a) $\mathrm{I}-S_{\theta}$ is small in $L^{\infty}$,

(b) $S_{\theta}^{*}=S_{\theta} \geq 0$,

(c) $d_{s, \theta} S_{\theta}=\left(-\sin \theta \frac{\partial}{\partial x}+\cos \theta \frac{\partial}{\partial y}\right) S_{\theta}=0$.

Proof. The existence of $E_{\mu}$ away from the circle $\mu \in S^{1} \subset \mathbb{C} P^{1}$ is a straight forward iteration argument involving estimates in $L^{\infty}$ on $E_{\mu}$ using $(A, \phi)$ small in $L^{1} \cap L^{\infty}$. We relegate the estimate as $\mu \rightarrow e^{i \theta} \in S^{1}$ to an appendix. Once the basic estimate are in place, proof of regularity and holomorphic dependence on $\mu$ is straightforward. We explicitly derive (a) in the appendix. To obtain (b), note that $\left(E_{\bar{u}^{-1}}\right)^{-1}=E_{\mu}^{*}$ due to the reality condition on $(A, \phi)$. Hence $\left(S_{\theta}^{-}\right)^{-1}=\left(S_{\theta}^{+}\right)^{*}$.

To obtain (c), notice that

$$
D_{s}\left(e^{i \theta}\right)=-i\left(d_{s, \theta}-\psi\right),
$$

where $\psi=-\psi^{*}=\sin \theta A_{x}-\cos \theta A_{y}+\phi$. Since $D_{s}(\mu) E_{\mu}=0$, it follows from the definition of $S^{+}$that $D_{s}\left(e^{i \theta}\right) S^{+}=0$, so $d_{s, \theta} S^{+}=\psi S^{+}$. But

$$
d_{s, \theta}\left(\left(S^{+}\right)^{*}\right)=\left(d_{s, \theta} S^{+}\right)^{*}=\left(\psi S^{+}\right)^{*}=\left(S^{+}\right)^{*} \psi^{*}=-\left(S^{+}\right)^{*} \psi .
$$

Now compute

$$
\begin{aligned}
d_{s, \theta} S & =d_{s, \theta}\left(\left(S^{+}\right)^{*} S^{+}\right)=\left(d_{s, \theta}\left(S^{+}\right)^{*}\right) S^{+}+\left(S^{+}\right)^{*} d_{s, \theta} S^{+} \\
& =-\left(S^{+}\right)^{*} \psi S^{+}+\left(S^{+}\right)^{*} \psi S^{+}=0
\end{aligned}
$$

and (c) follows.

Corollary 7.4. Assume $(A, \phi)$ is a smooth solution in $\mathbb{R}^{2} \times\left(T_{1}, T_{2}\right)$ and decays in spacial variables, and has a smooth continuous scattering data. Then

$$
0=\left(\frac{\partial}{\partial t}+\cos \theta \frac{\partial}{\partial x}+\sin \theta \frac{\partial}{\partial y}\right) S_{\theta} .
$$


The corollary assumes that the scattering theory is differentiable in $t$. Since $D_{t}(\mu)=D_{1}(\mu)+D_{2}(\mu)=\nabla_{t}+\mu \nabla_{z}+\mu^{-1} \nabla_{\bar{z}}$ has the property that $\left[D_{s}(\mu), D_{t}(\mu)\right]=0$, we conclude that $D_{t}(\mu) E_{\mu}=0$. Now the result follows by the same method as (c) in the proposition.

Corollary 7.5. If $(A, \phi)$ and $(\tilde{A}, \tilde{\phi})$ are gauge equivalent, then they have the same scattering data.

Proof. Suppose $(\tilde{A}, \tilde{\phi})$ is the gauge transformation of $(A, \phi)$ by a unitary map $u$. If $E_{\mu}(x, y, t)$ is the frame for $(A, \phi)$, then $u(x, y, t) E_{\mu}(x, y, t)$ is the frame for $(\tilde{A}, \tilde{\phi})$. Hence the limits $\tilde{S}_{\theta}^{+}=u S_{\theta}^{+}$and $\tilde{S}_{\theta}^{-}=u S_{\theta}^{-}$. But $u$ is unitary, so $\tilde{S}=S$.

Inverse scattering theory is simpler than scattering theory. To dispose the gauge ambiguity, we need to make a choice somewhere. We choose to do this at $\mu=1$. First we note that if we have initial scattering data $S_{\theta}(x, y)$ satisfying $d_{s, \theta} S_{\theta}(x, y)=0$, then the scattering data $S_{\theta}(x, y, t)$ for the solution at time $t$ should satisfy

$$
\frac{\partial S_{\theta}}{\partial t}+\cos \theta \frac{\partial S_{\theta}}{\partial x}+\sin \theta \frac{\partial S_{\theta}}{\partial y}=0
$$

Since $d_{s, \theta} S_{\theta}(x, y)=0$, there exists $s$ such that

$$
S_{\theta}(x, y)=s(x \cos \theta+y \sin \theta, \theta) .
$$

The linear evolution equation for $S_{\theta}(x, y, t)$ implies that

$$
S_{\theta}(x, y, t)=s(x \cos \theta+y \sin \theta-t, \theta) .
$$

The problem is now to write

$$
\left.S_{\mu}(x, y, t)=E_{\mu}^{-}(x, y, t)\right)^{-1} E_{\mu}^{+}(x, y, t), \quad \mu \in S^{1},
$$

where $E_{\mu}^{-}$extends holomorphically to $\mu$ inside the unit circle, $E_{\mu}^{+}$extends holomorphically outside the unit circle, and $E_{\mu}^{-}=\left(\left(E_{\bar{\mu}^{-1}}^{+}\right)^{*}\right)^{-1}$. We can always do this, and the ambiguity corresponds to the gauge transformations. The condition that $S_{1}(x, y, t)=\mathrm{I}$ is equivalent to the solution being 1regular. However, we choose a method of factoring which yields a unique solution for all scattering data. The inverse scattering was given in [21] by Villarroel and in [7] by Fokas-Ioannidou. We prove it using the Iwasawa loop group factorization theorem of Pressley and Segal [14]. Since $S_{\theta}$ is Hermitian symmetric and non-negative, there is a Hermitian symmetric matrix $P_{\mu}$ such that $S_{\theta}=P_{\mu}^{2}$, where $\mu=e^{i \theta}$. The Iwasawa loop group factorization of Pressley and Segal $[\mathbf{1 4}]$ says that we can factor

$$
P_{\mu}=U_{\mu} E_{\mu}^{+}
$$

uniquely such that $U_{\mu}$ is in $U(n), U_{1}=\mathrm{I}$, and $E_{\mu}^{+}$extends holomorphically to outside the unit circle. Set $E_{\mu}^{-}=\left(\left(E_{\bar{\mu}^{-1}}^{+}\right)^{*}\right)^{-1}$. Now $S_{\theta}=\left(E_{\mu}^{-}\right)^{-1} E_{\mu}^{+}$ when $\mu=e^{i \theta}$. Since $S_{\mu}$ is smooth, $E_{\mu}^{ \pm}$is smooth. 
Theorem 7.6 (Villarroel [21], Fokas-Ioannidou [7]). Let $S_{\theta}(x, y, t)=$ $s(x \cos \theta+y \sin \theta-t, \theta)$. Then the factorization described above

$$
S_{\theta}=\left(E_{\mu}^{-}\right)^{-1}(x, y, t) E_{\mu}^{+}(x, y, t),
$$

where $\mu=e^{i \theta}$ and $E_{\mu}^{ \pm}$can extend holomorphically to $|\mu|^{ \pm 1}>1$ yields smooth frame for a solution of the space-time monopole equation.

Proof. We need to show that $E_{\mu}^{ \pm}(x, y, t)$ generates a solution to the monopole equation. To do this, note by construction that $E_{\mu}^{ \pm}$is holomorphic in $|\mu|^{ \pm 1}>1$. The operator $d_{s, \theta}$ is a directional derivative, $d_{s, \theta} S=0$ and $S=\left(S^{-}\right)^{-1} S^{+}$, so

$$
0=d_{s, \theta} S=\left(d_{s, \theta}\left(\left(S^{-}\right)^{-1}\right)\right) S^{+}+\left(S^{-}\right)^{-1} d_{s, \theta} S^{+} .
$$

Thus we have

$$
\left(d_{s, \theta} S^{+}\right)\left(S^{+}\right)^{-1}=-S^{-} d_{s, \theta}\left(\left(S^{-}\right)^{-1}\right)=\left(d_{s, \theta} S^{-}\right)\left(S^{-}\right)^{-1} .
$$

Note that

$$
d_{s, \mu}=\frac{i\left(\mu-\mu^{-1}\right)}{2} \frac{\partial}{\partial x}+\frac{\mu+\mu^{-1}}{2} \frac{\partial}{\partial y}
$$

is the meromorphic extension of $d_{s, \theta}=d_{s, e^{i \theta}}$. So by meromorphic extension, we obtain the identity on $0<|\mu|<\infty$

$$
\left(d_{s, \mu} E_{\mu}^{+}\right)\left(E_{\mu}^{+}\right)^{-1}=\left(d_{s, \mu} E_{\mu}^{-}\right) E_{\mu}^{-1} .
$$

Since the left hand side is meromorphic in $|\mu|>1$ with a simple pole at $\infty$ and the right hand side is meromorphic on $|\mu|<1$ with a simple pole at 0 , both sides are meromorphic in $\mathbb{C}$ with simple poles at 0 and $\infty$. Let $E_{\mu}=E_{\mu}^{ \pm}$when $|\mu|^{ \pm}>1$. Then $E_{\bar{\mu}^{-1}}=\left(E_{\mu}^{*}\right)^{-1}$. It follows that

$$
\xi_{\mu}:=\left(d_{s, \mu} E_{\mu}\right) E_{\mu}^{-1}=\mu C_{1}+\mu^{-1} C_{-1}+C_{0}
$$

for some $C_{i}(x, y, t)$. But $d_{s, \bar{\mu}^{-1}}^{*}=d_{s, \mu}$ and $E_{\bar{\mu}^{-1}}=\left(E_{\mu}^{*}\right)^{-1}$, so $\xi_{\bar{\mu}^{-1}}^{*}=-\xi_{\mu}$. Hence $C_{-1}=-C_{1}^{*}$ and $C_{0}^{*}=-C_{0}$. Write $C_{1}=A_{z}, C_{-1}=A_{\bar{z}}$ and $C_{0}=\phi$; then we have

$$
\left(d_{s, \mu} E_{\mu}\right) E_{\mu}^{-1}=\mu A_{z}-\mu^{-1} A_{\bar{z}}+\phi
$$

where $A_{z}=-\left(A_{\bar{z}}\right)^{*}$ and $\phi^{*}=-\phi$. In other words, we have proved $\left(D_{1}(\mu)-\right.$ $\left.D_{2}(\mu)\right) E_{\mu}=0$.

The proof of the evolution equation, i.e., $D_{t}(\mu) E_{\mu}=0$, is similar and we do not carry it out here.

Of course, if we start with an initial condition which has only continuous scattering data, we will not necessarily obtain the same initial data by the inverse scattering transform, but obtain a gauge equivalent solution.

Corollary 7.7. Let $(A, \phi)$ be a solution of the space-time monopole equation rapidly decaying in the spacial variables. Assume in addition that the Chern vector $\vec{c}(\mu)=0$ for all $\mu \in \mathbb{C} \backslash S^{1}$. Assume also that the solution has continuous scattering data for all $t$. Then the solution obtained 
from the scattering data at $t=0$ agrees with the given solution up to gauge transformation.

Proof. We know from the assumption that $S_{\theta}(x, y, t)=s(x \cos \theta+$ $y \sin \theta-t, \theta)$, since $S_{\theta}$ is unique,

$$
S_{\theta}=\lim _{\mu \rightarrow e^{i \theta},|\mu|>1}\left(E_{\bar{\mu}^{-1}}\right)^{*} E_{\mu} .
$$

Hence the frame provides a factorization. This factorization is unique up to a unitary matrix $u=u(x, y, t)$. This unitary matrix gives a gauge transformation between the original solution and the one constructed by inverse scattering.

\section{1-soliton monopoles}

In addition to continuous scattering data, solutions of monopole equation may also have discrete scattering data. We first construct monopoles whose frames have one simple pole, and in later sections we construct frames with multiple poles and show how to combine them with continuous scattering data.

The building blocks of the discrete scattering data are one-solitons, which are easy to describe. We have the harmonic maps $\phi: \mathbb{R}^{2} \cup\{\infty\} \rightarrow$ $S U(n)$, which yield time-independent solutions to the Ward equation. Among these, we have one-unitons, which come from holomorphic maps into Grassmannians. We also have the Lorentz transformations of these stationary one unitons. This family makes up the one-solitions. It is somewhat more difficult to show that every one-soliton, defined in terms of a single pole for the frame, is of this type.

We need to use another gauge equivalent Lax pair to construct soliton solutions. If a monopole $(A, \phi)$ is $\lambda=0$ regular, then we can fix the gauge of $(2.9)$ at $\lambda=0$ to get

$$
\left[\lambda\left(\partial_{\xi}-\tilde{A}_{\xi}\right)-\partial_{y}, \lambda\left(\partial_{y}+2 \tilde{\phi}\right)-\partial_{\eta}\right]=0,
$$

where $\tilde{\phi}=-\tilde{A}_{y}$, and $\tilde{A}_{\eta}=0$. The above Lax pair is equivalent to

$$
\left.\left[\tau \partial_{y}-\partial_{\xi}+\tilde{A}_{\xi}\right), \tau \partial_{\eta}-\partial_{y}-2 \tilde{\phi}\right]=0 .
$$

(Here $\tau=\lambda^{-1}$.)

The spectral parameters $\mu, \lambda, \tau$ in Lax pairs (2.8), (2.9), and (8.1) are related by

$$
\mu=\frac{\lambda-i}{\lambda+i}, \quad \tau=\lambda^{-1}, \quad \text { so } \quad \tau=\lambda^{-1}=\frac{i(\mu-1)}{\mu+1} .
$$

The above discussion gives the following proposition (cf. [22]):

Proposition 8.1. Suppose there is a smooth $G L(n, \mathbb{C})$-valued $\psi(x, y$, $t, \tau)$ defined for $(x, y, t) \in \mathbb{R}^{2,1}$ and $\tau$ in an open subset of $\mathbb{C}$ such that

(1) $P:=\left(\tau \partial_{y} \psi-\partial_{\xi} \psi\right) \psi^{-1}$ and $Q:=\left(\tau \partial_{\eta} \psi-\partial_{y} \psi\right) \psi^{-1}$ are independent of $\tau$, 
(2) $\psi(x, y, t, \bar{\tau})^{*} \psi(x, y, t, \tau)=\mathrm{I}$.

Let $A$ be a connection and $\phi$ a Higgs field defined by $A_{\xi}=-P, A_{\eta}=0$, and $A_{y}=-\phi=-Q / 2$. Then $(A, \phi)$ is a solution of the monopole equation. Conversely, every solution of the monopole equation that is regular at $\lambda=0$ is gauge equivalent to a solution of this type.

Definition 8.2. A map $\psi$ that satisfies (1) and (2) of Proposition 8.1 is called a Ward frame if $P, Q$ decay in spacial infinity and $\psi(x, y, t, \infty)=\mathrm{I}$. A Ward frame is a Ward soliton frame if $\psi$ is rational in $\tau$.

Definition 8.3. A solution $(A, \phi)$ of the monopole equation is called a $k$-soliton if it is regular at $\mu=-1$ and has a monopole frame $E$ that is rational in $\mu$ with $k$ poles counted with multiplicity.

If $f: S^{2} \rightarrow G L(n, \mathbb{C})$ is rational with one simple pole at $\tau=\alpha$, then it can be checked that (cf. [19]) $f$ must be of the form

$$
g_{\alpha, \pi}(\tau)=\mathrm{I}+\frac{\alpha-\bar{\alpha}}{\tau-\alpha} \pi^{\perp}
$$

where $\pi^{\perp}=\mathrm{I}-\pi$ and $\pi$ is a Hermitian projection $\pi$ of $\mathbb{C}^{n}$. We identify the space of rank $m$ Hermitian projections of $\mathbb{C}^{n}$ as $\operatorname{Gr}\left(m, \mathbb{C}^{n}\right)$ via the map $\pi \mapsto \operatorname{Im}(\pi)$. So a Ward 1-soliton frame must be of the form $g_{\alpha, \pi(x, y, t)}(\tau)=$ $\mathrm{I}+\frac{\alpha-\bar{\alpha}}{\tau-\alpha} \pi^{\perp}(x, y, t)$ for some constant $\alpha \in \mathbb{C} \backslash \mathbb{R}$ and $\pi: S^{2} \times \mathbb{R} \rightarrow \operatorname{Gr}\left(k, \mathbb{C}^{n}\right)$. Note that $\left(\tau \partial_{y} \psi-\partial_{\xi} \psi\right) \psi^{-1}$ and $\left(\tau \partial_{\eta} \psi-\partial_{y} \psi\right) \psi^{-1}$ are independent of $\tau$ if and only if the residue at $\tau=\alpha$ is zero. This implies

Proposition 8.4. Given $\alpha \in \mathbb{C} \backslash \mathbb{R}$ a constant and $\pi: \mathbb{R}^{2,1} \rightarrow \operatorname{Gr}\left(k, \mathbb{C}^{n}\right)$ a smooth map, then $g_{\alpha, \pi}(\tau)=\mathrm{I}+\frac{\alpha-\bar{\alpha}}{\tau-\alpha} \pi^{\perp}$ is a Ward soliton frame if and only if

$$
\left\{\begin{array}{l}
\left(\alpha \partial_{y} \pi-\partial_{\xi} \pi\right) \pi=0 \\
\left(\alpha \partial_{\eta} \pi-\partial_{y} \pi\right) \pi=0 .
\end{array}\right.
$$

Moreover, if $\pi$ is a solution of (8.2), then there exists a holomorphic map $\pi_{0}: S^{2} \rightarrow \operatorname{Gr}\left(k, \mathbb{C}^{n}\right)$ such that $\pi(x, y, t)=\pi_{0}\left(y+\alpha \xi+\alpha^{-1} \eta\right)$.

Note that if $\alpha= \pm i$, then $x+\alpha \xi+\alpha^{-1} \eta=y \pm i x$ and $\pi(x, y, t)=$ $\pi_{0}(y \pm i x)$. So the 1 -soliton $g_{ \pm i, \pi}$ is a 1-uniton harmonic map.

The $S O(2,1)$-actions described in Section 4 of 1-unitons are 1-soliton monopoles. In fact, we have

Proposition 8.5. Given $\alpha=r e^{i \theta} \in \mathbb{C} \backslash \mathbb{R}$, let $e^{s}=r, e^{c}=\csc \theta+\cot \theta=$ $\cot (\theta / 2)$, and $h=T(c) R(-\pi / 2) T(s) \in S O(2,1)$, where $T(c)$ and $R(\theta)$ are 1-parameter subgroups of $S O(2,1)$ defined in Section 4 . Let $\pi_{0}: S^{2} \rightarrow$ $\operatorname{Gr}\left(k, \mathbb{C}^{n}\right)$ be a holomorphic map. Then the action of $h$ on 1-uniton frame $g_{i, \pi_{0}}$ gives rise to a monopole solution that is gauge equivalent to the 1-soliton given by $g_{\alpha, \pi_{0}}$. In other words, all 1-solitons monopoles are obtained from the action of $S O(2,1)$ on 1-unitons up to gauge equivalence. 
Proof. Recall that $\lambda=\tau^{-1}$, and

$$
F(x, y, t, \lambda)=g_{i, \pi_{0}(y+i x)}\left(\lambda^{-1}\right)
$$

is a solution for the linear system $(2.11)(\operatorname{Lax}$ pair in $\lambda)$. Let $(\tilde{x}, \tilde{y}, \tilde{t})^{t}=$ $h(x, y, t)^{t}$. A computation gives

$$
\tilde{y}+i \tilde{x}=\frac{i}{\sin \theta}\left(y+\alpha \xi+\alpha^{-1} \eta\right)
$$

Let $\tilde{\lambda}=h * \lambda$. Then $\tilde{\lambda}=\frac{e^{c}\left(e^{s} \lambda-1\right)}{e^{s} \lambda+1}$, so the pole of this expression is when $\tilde{\lambda}=-i$, i.e, when $\frac{e^{c}\left(e^{s} \lambda-1\right)}{e^{s} \lambda+1}=-i$. But $r=e^{s}$ and $e^{c}=\cot (\theta / 2)$, so the pole is at $\lambda=\alpha^{-1}$. This shows that $h \cdot F$ has one simple pole at $\lambda=\alpha^{-1}$. Note that $h \cdot F$ is equal to

$$
\begin{aligned}
(h \cdot F)(x, y, t, \lambda) & =g_{i, \pi_{0}(\tilde{y}+i \tilde{x})}\left((\sigma(h) * \lambda)^{-1}\right) \\
& =\pi_{0}(\tilde{y}+i \tilde{x})-e^{i \theta} \frac{\lambda^{-1}-\bar{\alpha}}{\lambda^{-1}-\alpha} \pi_{0}^{\perp}(\tilde{y}+i \tilde{x}) \\
& =\left(\pi(x, y, t)-e^{i \theta} \pi^{\perp}(x, y, t)\right) g_{\alpha, \pi(x, y, t)}(\tau),
\end{aligned}
$$

where $\pi(x, y, t)=\pi_{1}\left(y+\alpha \xi+\alpha^{-1} \eta\right)$ and $\pi_{1}(z)=\pi_{0}(i z / \sin \theta)$ is holomorphic. So $h \cdot F$ is gauge equivalent to the 1 -soliton $g_{\alpha, \pi}$. Note since $\pi_{0}: S^{2} \rightarrow$ $\operatorname{Gr}\left(k, \mathbb{C}^{n}\right)$ is smooth and $h \in S O(2,1)$, the monopole given by $g_{\alpha, \pi}$ decays at spacial variables.

\section{Bäcklund transformations and construction of soliton monopoles}

Multisolitons with simple poles were constructed by Ward [22]. Ward, Ioannidou, and Anand $([\mathbf{2 4}, \mathbf{1 0}, \mathbf{2}])$ derived methods for computing solitons which have poles with higher multiplicities. These multisolitons have dramatic physical properties. We give here a brief description of a method of "superposing" solitons, which is closely related to the permutability formula for Bäcklund transformations. This technique allowed Dai and Terng [6] to construct solitons with an arbitrary number of poles with arbitrary multiplicities.

Intuitively, the permutability formula is based on factoring frames. Given the frames of two solutions $\psi_{1}$ and $\psi_{2}$ with singularities at different sets $S_{1}$ and $S_{2}, S_{1} \cap S_{2}=\emptyset$, in $\mathbb{C} \cup\{\infty\}$, we write

$$
\psi_{3}=\tilde{\psi}_{1} \psi_{2}=\tilde{\psi}_{2} \psi_{1}
$$

(i.e., factor $\psi_{1} \psi_{2}^{-1}=\tilde{\psi}_{2}^{-1} \tilde{\psi}_{1}$ ). Here $\psi_{3}$ has the singularities at $S_{1} \cup S_{2}$, and $\psi_{j}$ and $\tilde{\psi}_{j}$ have the same singular set $S_{j}$. It is not difficult to see that $\psi_{3}$ is a frame for a solution when $\psi_{1}$ and $\psi_{2}$ are. The details of allowing limiting case where $S_{1} \rightarrow S_{2}$ yield the interesting but complex solitons. The converse of factoring solutions is also true, but not completely straightforward. We now go to the details. 
Theorem 9.1 (Algebraic Bäcklund transformation). Let $\psi(x, y, t, \tau)$ be a Ward frame with $P=\left(\tau \partial_{y} \psi-\partial_{\xi} \psi\right) \psi^{-1}$ and $Q=\left(\tau \partial_{\eta} \psi-\partial_{y} \psi\right) \psi^{-1}$, and $g_{\alpha, \pi}$ a 1-soliton Ward frame. Suppose $\psi$ is holomorphic and nondegenerate at $\tau=\alpha$. Let $\tilde{\pi}(x, y, t)$ denote the Hermitian projection of $\mathbb{C}^{n}$ onto $\psi(x, y, t, \alpha)(\operatorname{Im}(\pi(x, y, t))$. Then

(1) $\tilde{\psi}(x, y, t, \tau)=g_{\alpha, \tilde{\pi}(x, y, t)}(\tau) \psi(x, y, t, \tau) g_{\alpha, \pi(x, y, t)}(\tau)^{-1}$ is holomorphic and non-degenerate at $\tau=\alpha, \bar{\alpha}$,

(2) $\psi_{1}=g_{\alpha, \tilde{\pi}} \psi=\tilde{\psi} g_{\alpha, \pi}$ is again a Ward frame such that

$$
\left\{\begin{array}{l}
\left(\tau \partial_{y} \psi_{1}-\partial_{\xi} \psi_{1}\right) \psi_{1}^{-1}=\tilde{P} \\
\left(\tau \partial_{\eta} \psi_{1}-\partial_{y} \psi_{1}\right) \psi_{1}^{-1}=\tilde{Q},
\end{array}\right.
$$

where $\tilde{P}=P+(\bar{\alpha}-\alpha) \partial_{y} \tilde{\pi}$ and $\left.\tilde{Q}=Q+(\bar{\alpha}-\alpha) \partial_{\eta} \tilde{\pi}\right)$.

We will use $g_{\alpha, \pi} * \psi$ to denote $\psi_{1}$.

Proof. We give a sketch the proof. Statement (1) can be proved by computing the residue of $\tilde{\psi}$ at $\tau=\alpha$ and show that it is zero. We use Proposition 8.1 to prove (2). Set $\tilde{g}=g_{\alpha, \tilde{\pi}}$. Since $D=\left(\tau \partial_{y}-\partial_{\xi}\right)$ is a derivation, we have

$$
\left(D \psi_{1}\right) \psi_{1}^{-1}=(D \tilde{g}) \tilde{g}^{-1}+\tilde{g}(D \psi) \psi^{-1} \tilde{g}^{-1}=(D \tilde{g}) \tilde{g}^{-1}+\tilde{g} P \tilde{g}^{-1},
$$

so it is holomorphic for $\tau \in \mathbb{C} \backslash\{\alpha\}$ and has a simple pole at $\tau=\alpha$. But $\psi_{1}$ is also equal to $\tilde{\psi} g$ (here $g=g_{\alpha, \pi}$ is a 1 -soliton Ward frame), so

$$
\left(D \psi_{1}\right) \psi_{1}^{-1}=(D \tilde{\psi}) \tilde{\psi}^{-1}+\tilde{\psi}(D g) g^{-1} \tilde{\psi}^{-1} .
$$

But $(D g) g^{-1}$ is independent of $\tau$ and $\tilde{\psi}$ is holomorphic and non-degenerate at $\tau=\alpha, \bar{\alpha}$, hence the RHS is holomorphic at $\tau=\alpha$. So $\left(D \psi_{1}\right) \psi_{1}^{-1}$ is holomorphic in $\mathbb{C}$. But the residue of $\left(D \psi_{1}\right) \psi_{1}^{-1}$ at $\tau=\infty$ is also zero. Hence it must be independent of $\tau$. Similar argument implies that $\left(\tau \partial_{\eta} \psi-\partial_{x} \psi\right) \psi^{-1}$ is also independent of $\tau$, so by Proposition 8.1, $\psi_{1}$ is a Ward frame.

Set $\tilde{P}=\left(D \psi_{1}\right) \psi_{1}^{-1}=(D \tilde{g}) \tilde{g}^{-1}+\tilde{g} P \tilde{g}^{-1}$. Evaluate the residue at $\tau=\infty$ to get $\tilde{P}=\tilde{P}+(\bar{\alpha}-\alpha) \partial_{y} \tilde{\pi}$. Similarly, we get the formula for $\tilde{Q}$.

Since $P, Q$ decay at spacial infinity, $\mathrm{I}-\psi(\ldots, \alpha)$ decays at spacial infinity. But $g_{\alpha, \pi}$ is a 1 -soliton monopole frame, so $\partial_{x} \pi, \partial_{\eta} \pi$ also decay at spacial infinity. Hence $\tilde{P}, \tilde{Q}$ decays at spacial infinity.

\section{$k$-soliton monopole frames with only simple poles}

Let $\alpha_{1}, \ldots, \alpha_{k}$ be distinct complex numbers and $\operatorname{Im}\left(\alpha_{j}\right)>0$ for all $1 \leq j \leq k, \pi_{j}^{0}: S^{2} \rightarrow \operatorname{Gr}\left(k_{j}, \mathbb{C}^{n}\right)$ holomorphic maps, and $\pi_{j}(x, y, t)=$ $\pi_{j}^{0}\left(y+\alpha_{j} \xi+\alpha_{j}^{-1} \eta\right)$. Then $g_{\alpha_{j}, \pi_{j}}$ is a 1-soliton Ward frame. Apply the Algebraic BT (Theorem 9.1) repeatedly as follows: Set $\psi_{1}=g_{\alpha_{1}, \pi_{1}}$, and define $\psi_{j}$ inductively by $\psi_{j}=g_{\alpha_{j}, \pi_{j}} * \psi_{j-1}$ for $2 \leq j \leq k$. Then $\psi_{k}$ is a $k$-soliton Ward frame with $k$ simple distinct poles at $\alpha_{1}, \ldots, \alpha_{k}$. These are the same soliton Ward frames constructed by Ward using the solution to the Riemann-Hilbert problem. 


\section{$k$-soliton monopole frames with pole data $(\alpha, k)$}

Ward's limiting construction is as follows: Let $f_{0}, f_{1}$ be rational maps from $\mathbb{C}$ to $\mathbb{C}^{2}$, and $\pi_{1, \epsilon}$ and $\pi_{2, \epsilon}$ the projections of $\mathbb{C}^{2}$ onto the complex line spanned by $f_{0}\left(w_{i+\epsilon}\right)+\epsilon f_{1}\left(w_{i+\epsilon}\right)$ and $f_{0}\left(w_{i-\epsilon}\right)-\epsilon f_{1}\left(w_{i-\epsilon}\right)$ respectively, where $w_{i \pm \epsilon}=y+(i \pm \epsilon) \xi+(i \pm \epsilon)^{-1} \eta$. Ward showed that

$$
\psi=\lim _{\epsilon \rightarrow 0} g_{i-\epsilon, \pi_{2, \epsilon}} * g_{i+\epsilon, \pi_{1, \epsilon}}
$$

is a 2-soliton Ward frame with a double pole at $\tau=i$ and is not stationary.

Since the algebraic BT is easy to compute, Ward's limiting method can be calculated systematically as follows (for detail see the paper by Dai and Terng [6]): Let $\alpha_{\epsilon}=\alpha+\epsilon, a_{j}: \mathbb{C} \rightarrow \mathbb{C}^{n}$ be rational maps, and $f_{j, \epsilon}=$ $a_{0}+a_{1} \epsilon+\ldots+a_{j-1} \epsilon^{j-1}$. Let $\pi_{j, \epsilon}(x, y, t)$ denote the Hermitian projection of $\mathbb{C}^{n}$ onto the complex line spanned by $f_{j, \epsilon}\left(y+\alpha_{\epsilon} \xi+\alpha_{\epsilon}^{-1} \eta\right)$. Set $\psi_{1}=$ $\lim _{\epsilon \rightarrow 0} g_{\alpha_{\epsilon}, \pi_{1, \epsilon}}$, which is a 1-soliton frame $g_{\alpha, \pi}$ (here $\pi$ is the projection onto $\left.\mathbb{C} a_{0}\left(y+\alpha \xi+\alpha^{-1} \eta\right)\right)$. Define $\psi_{k}$ inductively by $\psi_{k}=\lim _{\epsilon \rightarrow 0} g_{\alpha_{\epsilon}, \pi_{k, \epsilon}} * \psi_{k-1}$. Then $\psi_{k}$ is a $k$-soliton frame with pole data $(\alpha, k)$ (i.e., $\psi_{k}$ has a single pole at $\tau=\alpha$ with multiplicity $k$ ). Note that $\psi_{k}$ depend on $k$ holomorphic maps from $\mathbb{C} P^{1}$ to $\cup_{i=1}^{n-1} \operatorname{Gr}\left(i, \mathbb{C}^{n}\right)$.

\section{Soliton frames with arbitrary pole data}

To get Ward soliton frames with arbitrary pole data, we need a more general BT for adding a $k$-soliton with pole data $(\alpha, k)$ to an existing Ward frame (cf. [6]):

TheOREM 9.2 (Adding a $k$-soliton with pole data $(\alpha, k)$ ). Suppose $\psi$ is a Ward frame that is holomorphic and non-degenerate at $\tau=\alpha, \bar{\alpha}$, and $\phi$ a $k$-soliton monopole frame with pole data $(\alpha, k)$. Then there exist unique $\tilde{\phi}$ and $\tilde{\psi}$ such that $\tilde{\phi} \psi=\tilde{\psi} \phi, \tilde{\phi}$ has pole data $(\alpha, k)$, and $\tilde{\psi}$ is holomorphic and non-degenerate at $\tau=\alpha, \bar{\alpha}$. Moreover, $\hat{\psi}=\tilde{\phi} \psi=\tilde{\psi} \phi$ is again a Ward frame and $\tilde{\phi}$ and $\tilde{\psi}$ are constructed algebraically.

As a consequence, we see that the two BTs and the limiting method give rise to Ward soliton frames with arbitrary pole data. The following theorem was proved in $[6]$.

TheOrem $9.3([\mathbf{6}])$. Algebraic BTs, adding $k$-soliton BTs, and the limiting method produce all soliton monopoles up to gauge equivalence.

If $\pi_{0}: S^{2} \rightarrow \operatorname{Gr}\left(m, \mathbb{C}^{n}\right)$ is holomorphic, then the limit of the 1-soliton frame,

$$
\lim _{\|(x, y)\| \rightarrow \infty} \mathrm{I}+\frac{\alpha-\bar{\alpha}}{\tau-\alpha} \pi_{0}^{\perp}\left(y+\alpha \xi+\alpha^{-1} \eta\right)=\mathrm{I}+\frac{\alpha-\bar{\alpha}}{\tau-\alpha} \pi_{0}^{\perp}(\infty)
$$

exists as $(x, y)$ tends to infinity and is independent of $t$. In other words, the Ward soliton frame tends to a fixed rational map $h(\tau)$ at spacial infinity and is independent of time. It can be checked easily that this property is preserved under the Algebraic BT and limiting method. Hence we have 
Proposition 9.4. If $\psi$ is a Ward soliton frame, then $\lim _{\|(x, y)\| \rightarrow \infty} \psi(x$, $y, t, \tau)$ exists and is independent of $t$.

\section{Monopoles with both continuous and discrete scattering data}

The Lax pair (2.9) of the monopole equation is equivalent to

$$
\left[\tau\left(\nabla_{y}-\phi\right)-\nabla_{\xi}, \tau \nabla_{\eta}-\nabla_{y}-\phi\right]=0 .
$$

The linear system associated to this Lax pair is

$$
\left\{\begin{array}{l}
\left(\tau \partial_{y}-\partial_{\xi}\right) \psi=\left(\tau A_{y}+\tau \phi-A_{\xi}\right) \psi, \\
\left(\tau \partial_{\eta}-\partial_{y}\right) \psi=\left(\tau A_{\eta}+\phi-A_{y}\right) \psi .
\end{array}\right.
$$

The Algebraic BT theorem for the monopole equation can be proved the same way as for the Ward equation. We only state the result:

TheOREm 10.1 (Algebraic BT for Monopoles). Suppose $\alpha \in \mathbb{C} \backslash \mathbb{R}$ is a constant, and $\psi$ is a frame of the monopole solution $(A, \phi)$ (i.e., solution of (10.2)), and $\psi(x, y, t, \tau)$ is holomorphic and non-degenerate at $\tau=\alpha$. Let $g_{\alpha, \pi}$ be a 1-soliton Ward frame, $\tilde{\pi}(x, y, t)$ the Hermitian projection onto $\psi(x, y, t, \alpha)(\operatorname{Im} \pi(x, y, t))$, and

$$
\tilde{\psi}=g_{\alpha, \tilde{\pi}} \psi g_{\alpha, \pi}^{-1} .
$$

Then

(1) $\tilde{\psi}$ is holomorphic and non-degenerate at $\tau=\alpha$,

(2) $\psi_{1}=g_{\alpha, \tilde{\pi}} \psi=\tilde{\psi} g_{\alpha, \pi}$ is a frame for (10.1) with $\tilde{A}, \tilde{\phi}$ given by

$$
\left\{\begin{array}{l}
\tilde{A}_{\eta}=A_{\eta}, \\
\tilde{A}_{\xi}=\left(1-\frac{\bar{a}}{\alpha}\right)\left(\partial_{\xi} \tilde{\pi}\right) h+h^{-1} A_{\xi} h, \\
\tilde{A}_{y}+\tilde{\phi}=A_{y}+\phi \\
\tilde{A}_{y}-\tilde{\phi}=\left(1-\frac{\bar{\alpha}}{\alpha}\right)\left(\partial_{y} \tilde{\pi}\right) h+h^{-1}\left(A_{y}-\phi\right) h,
\end{array}\right.
$$

where $h=\tilde{\pi}+\frac{\alpha}{\bar{\alpha}} \tilde{\pi}^{\perp}$.

Theorem 9.2 works for normalized monopole frames too.

Suppose $k: \mathbb{C} \rightarrow G L(n, \mathbb{C})$ is meromorphic, $k(\infty)=\mathrm{I}$, and $k(\bar{\tau})^{*} k(\tau)$ $=\mathrm{I}$. Then

satisfies

$$
\tilde{k}(x, y, t, \tau)=k\left(y+\tau \xi+\tau^{-1} \eta\right)
$$

$$
\left(\tau \partial_{y}-\partial_{\xi}\right) \tilde{k}=0, \quad\left(\tau \partial_{\eta}-\partial_{y}\right) \tilde{k}=0 .
$$

So if $\psi$ is a solution of (10.2) for the monopole solution $(A, \phi)$, then so is $\psi \tilde{k}$. However, if $\lim _{\|(x, y)\| \rightarrow \infty} \tilde{k}(x, y, t, \tau)$ exists and is independent of $t$, then $k$ must be the constant map I. Hence we can use this condition to normalize frames to get a unique one:

Definition 10.2. A solution $\psi$ of (10.2) for the monopole $(A, \phi)$ is called the normalized monopole frame if 
(1) $\psi(x, y, t, \bar{\tau})^{*} \psi(x, y, t, \tau)=\mathrm{I}$,

(2) there exists a map $h(\tau)$ such that $\lim _{\|(x, y)\| \rightarrow \infty} \psi(x, y, t, \tau)=h(\tau)$ exists and is independent of $t$.

By Proposition 9.4, a Ward soliton frame is a normalized monopole frame. By the Inverse scattering Theorem 7.6, given a smooth map $s$ : $\mathbb{R} \times S^{1} \rightarrow G L(n, \mathbb{C})$ such that $\mathrm{I}-s\left(\cdot, e^{i \theta}\right)$ decays for each $\theta$ and $s^{*}=s \geq 0$, then there exists a solution $E(x, y, t, \mu)$ of the linear system (2.10) such that

$$
\left(\left(E^{-}\right)^{-1} E^{+}\right)\left(x, y, 0, e^{i \theta}\right)=s\left(x \cos \theta+y \sin \theta, e^{i \theta}\right)
$$

$E(x, y, t, \mu) \rightarrow \mathrm{I}$ as $\|(x, y)\| \rightarrow \infty$, and $E$ is holomorphic in $|\mu| \neq 1$. Such $E$ is a normalized monopole frame with only continuous scattering data.

If we apply Algebraic BTs and General Algebraic BTs repeatedly to a normalized monopole frame with only continuous scattering data, then we obtain normalized monopoles frames with both continuous and discrete scattering data. So we get

THEOREM 10.3. Let $s: \mathbb{R} \times S^{1} \rightarrow G L(n, \mathbb{C})$ be a smooth map such that $\mathrm{I}-s\left(\cdot, e^{i \theta}\right)$ decays for each $\theta$ and $s^{*}=s \geq 0$, and $\phi_{j}$ a normalized soliton monopole frame with pole data $\left(\alpha_{j}, n_{j}\right)$ for $j=1, \ldots, k$. Then there is a unique normalized monopole frame $E(x, y, t, \mu)$ such that

(1) $E$ is holomorphic for $\mu \in \mathbb{C} \backslash\left(S^{1} \cup\left\{\alpha_{1}, \ldots, \alpha_{k}\right\}\right)$, has poles at $\alpha_{j}$ with multiplicity $n_{j}$, and

$$
E_{ \pm}\left(x, y, t, e^{i \theta}\right)=\lim _{|\mu|^{ \pm 1}<1, \mu \rightarrow e^{i \theta}} E(x, y, t, \mu)
$$

exist and are smooth,

(2) $\left(E_{-}^{-1} E_{+}\right)\left(x, y, t, e^{i \theta}\right)=s\left(x \cos \theta+y \sin \theta-t, e^{i \theta}\right)$ is the continuous scattering data of $M$,

(3) $E \phi_{j}^{-1}$ is holomorphic and non-degenerate at $\mu=\alpha_{j}$ for $1 \leq j \leq k$.

Below we want to prove that all normalized monopole frames with only finitely many poles and a jump across $S^{1}$ are constructed by the above method.

First we need to recall a factorization result. Let $\mathcal{D}$ denote the group of $f: S^{2}=\mathbb{C} \cup\{\infty\} \rightarrow G L(n, \mathbb{C})$ that satisfies the following conditions:

(1) $f\left(\bar{\mu}^{-1}\right)^{*} f(\mu)=\mathrm{I}$,

(2) $f_{ \pm}\left(e^{i \theta}\right):=\lim _{|\mu|^{ \pm}<1, \mu \rightarrow e^{i \theta}} f(\mu)$ exist and are smooth,

(3) $f$ is holomorphic in $S^{2} \backslash S^{1}$ except with possible finitely many poles.

The following results were proved in $[\mathbf{1 8}]$ :

(a) Given $f \in \mathcal{D}$, then there exist uniquely $h_{i}, g_{i} \in \mathcal{D}$ such that $f=$ $h_{1} g_{1}=g_{2} h_{2}, f_{i}$ is holomorphic in $S^{2} \backslash S^{1}, g_{i}$ are rational maps, and $g_{i}(-1)=\mathrm{I}$ for $i=1,2$.

(b) If $f \in \mathcal{D}$ has a simple pole at $\alpha$, then there is a unique projection $\pi$ such that $f g_{\alpha, \pi}^{-1}$ is holomorphic and non-degenerate at $\alpha$. 
Note that if $E$ is a normalized monopole frame then $E(x, y, t, \cdot) \in \mathcal{D}$. Moreover,

(i) $E$ is a soliton frame if and only if $E$ is rational in $\mu$,

(ii) $E$ has only continuous scattering data if and only if $E(x, y, t, \cdot)$ is holomorphic in $S^{2} \backslash S^{1}$.

For general normalized monopole frames with both poles and jumps across $S^{1}$, we have

THEOREM 10.4 (Subtracting 1-soliton). Suppose $\psi$ is a normalized monopole frame, and $\psi$ has a simple pole at $\tau=\alpha$ (may have other singularities as well). Then there exist unique $\tilde{\psi}, \psi_{1}$ and smooth $\pi, \tilde{\pi}: \mathbb{R}^{2,1} \rightarrow \operatorname{Gr}\left(k, \mathbb{C}^{n}\right)$ such that

(i) $\psi=\tilde{\psi} g_{\alpha, \pi}=g_{\alpha, \tilde{\pi}} \psi_{1}$,

(ii) $\tilde{\psi}$ and $\psi_{1}$ are holomorphic and non-degenerate at $\tau=\alpha, \bar{\alpha}$,

(iii) $g_{\alpha, \pi}$ is a normalized 1-soliton monopole frame, and $\psi_{1}$ is a normalized monopole frame.

Proof. Statements (i) and (ii) follow from known results stated just before the theorem. To prove (iii) we use residue calculus. Set $D=\tau \partial_{y}-\partial_{\xi}$, and let $g:=g_{\alpha, \pi}, \tilde{g}:=g_{\alpha, \tilde{\pi}}$. Then

$$
(D \psi) \psi^{-1}=(D \tilde{\psi}) \tilde{\psi}^{-1}+\tilde{\psi}(D g) g^{-1} \tilde{\psi}
$$

Since the LHS is independent of $\tau$, the residue of the RHS at $\tau=\alpha$ must be zero, so

$$
-\tilde{\psi}(\ldots, \alpha)\left(\alpha \partial_{y} \pi-\partial_{\xi} \pi\right) \pi \tilde{\psi}(\ldots, \alpha)^{-1}=0 .
$$

But $\tilde{\psi}(\ldots, \alpha)$ is non-degenerate, hence $\left(\alpha \partial_{y} \pi-\partial_{\xi} \pi\right) \pi=0$. Similarly, calculate the residue at $\tau=\alpha$ in the expression $\left(\tau \partial_{\eta} \psi-\partial_{y} \psi\right) \psi^{-1}$ to get $\left(\alpha \partial_{\eta} \pi-\partial_{y} \pi\right) \pi=0$. By Proposition 8.4, $g_{\alpha, \pi}$ is a 1-soliton monopole frame. Since $\psi_{1}=\tilde{g}^{-1} \psi$

$$
\left(D \psi_{1}\right) \psi_{1}^{-1}=-\tilde{g}^{-1} D \tilde{g}+\tilde{g}^{-1}(D \psi) \psi^{-1} \tilde{g} .
$$

The LHS is holomorphic at $\tau=\alpha, \bar{\alpha}$ and the RHS is holomorphic for all $\tau \neq \alpha, \bar{\alpha}$. So $\left(D \psi_{1}\right) \psi_{1}^{-1}$ is a degree one polynomial in $\tau$. Similarly, $\left(\tau \partial_{\eta} \psi_{1}-\right.$ $\left.\partial_{x} \psi_{1}\right) \psi_{1}^{-1}$ is a degree one polynomial in $\tau$. By Proposition 8.1, $\psi_{1}$ is a monopole frame.

\section{Similar argument gives}

THEOREM 10.5 (Subtracting a soliton with pole data $(\alpha, k)$ ). Suppose $\psi$ is a normalized monopole frame, and $\psi$ has a pole at $\tau=\alpha$ with multiplicity $k$ (may have other singularities as well). Then there exists a unique $k$-soliton monopole frame $g$ with pole data $(\alpha, k)$, a normalized monopole frame $\psi_{1}$, and maps $\tilde{\psi}$ and $\tilde{g}$ such that $\psi_{1}$ and $\tilde{\psi}$ are holomorphic and non-degenerate at $\tau=\alpha, \bar{\alpha}$ and $\psi=\tilde{\psi} g=\tilde{g} \psi_{1}$. 
A consequence of the above two theorems is that every normalized monopole frame with continuous scattering data and finitely many poles can be obtained from Theorem 10.3.

\section{Appendix: Existence of Continuous Scattering Data for Small Solutions}

The over-all details of the scattering data described in Sections 7 and 10 where we find solutions with a combination of a jump across the unit circle and point (singularities) measures do fit into the general scheme proposed by Beals and Coifman [4], [5]. Beals and Coifman point out that the Selfdual Yang-Mills equations have local scattering data more like the AKNS scattering problem than either type of KP, and Fokas and Ioannidou point out that the Ward equation inherits this similarity. Existence of solutions which have combinations of the two types of scattering data follows from the existence of the inverse scattering transform and the process of adding discrete measures by the described Bäcklund transforms. Papers of Fokas and Ioannidou [7] and Villarroel [21] discuss the existence of the scattering and inverse scattering transforms.

We find that, as in the AKNS models, small data leads to scattering data which consists only of the continuous part. For simplicity, we assume all the data is rapidly decaying and lies in the Schwartz space. Recall that the spacial operator is

$$
D_{s}(\mu)=D_{1}(\mu)-D_{2}(\mu)=\frac{\left(\mu-\mu^{-1}\right)}{2} \nabla_{x}-i \frac{\left(\mu+\mu^{-1}\right)}{2} \nabla_{y}-i \phi .
$$

THEOREM 11.1. Assume that there exists a gauge transformation such that in the given gauge $(A, \phi)$ is small in $W^{2,1}$. Then the chern vector $c(\mu)$ is zero for all $\mu \in C \cup\{\infty\} \backslash S^{1}$. Furthermore there exists continuous scattering data $S_{\theta}=I+G_{\theta}$ where $G_{\theta}$ is small in $L_{(x, y, t)}^{\infty}$.

Corollary 11.2. Given initial data $(A, \phi)$ which is small in $W^{2,1}$, there exist global in time solutions of the space-time monopole problem with this initial data. These solutions are unique up to gauge transformation.

First we explain how the corollary follows from the theorem. The existence of global solutions follows from the existence of scattering data which is purely continuous for the initial data, the known flow of the scattering data under time, and the existence of the inverse scattering transform. Because the scattering data remains small, the solution remains small in time. Suppose there is a time $t_{\theta}$ at which uniqueness fails. Since we presume the solution to be continuous in time, the second solution is small for a short time near $t_{\theta}$. Hence it has scattering data, and must be identified with the solution constructed by inverse scattering data by a gauge transformation.

To prove the theorem, we note that the proof is rather standard away from the unit circle, although it will follow from the proof we give near the unit circle as well. The difficulty is to prove that the limits exist as 
$|\mu| \rightarrow 1$. To do this, use the combination of Lorentz and fractional linear transformation so that $\mu$ is pure imaginary. Now let

$$
\tau=\left(\mu+\mu^{-1}\right) /\left(\mu-\mu^{-1}\right),
$$

so $\tau<1$ is real. We assume $|\mu|<1$, so $\tau<0$. For $|\mu|>1$, then the reality condition implies that $E_{\mu}=\left(\left(E_{\bar{\mu}^{-1}}\right)^{*}\right)^{-1}$. The equation for the frame now reads:

$$
\left(\partial_{x}-i \tau \partial_{y}\right) E_{\tau}=G_{\tau}
$$

where

$$
G_{\tau}(x, y, t)=A_{\tau}(x, y, t) E_{\tau}(x, y, t), \quad A_{\tau}=A_{x}-i \tau A_{y}(x, t)-\sqrt{1-\tau^{2}} \Phi .
$$

As is usual with solutions with small data, we solve by iteration. Let $E_{\tau}=\mathrm{I}+\sum_{j \geq 1} Q_{j, \tau}$. Set $Q_{0, \tau}=\mathrm{I}, Q_{j, \tau}(-\infty, y)=0$, and define $Q_{j, \tau}$ iteratively by

$$
\left(\partial_{x}-i \tau \partial_{y}\right) Q_{j, \tau}=G_{j-1, \tau}=A_{\tau} Q_{j-1, \tau}
$$

Here $A_{\tau}$ is as above.

We now take the Fourier transform in the $y$ variable alone, and denote the transform in $y$ only of an expression $Q$ by $\hat{Q}$. Let $\zeta$ be the transform variable of $y$. We have

$$
\partial_{x} \hat{Q}_{j, \tau}-\zeta \tau \hat{Q}_{j, \tau}=\hat{G}_{j-1, \tau}
$$

But this ODE can be solved explicitly:

$$
\hat{Q}_{j, \tau}(x, \zeta, t)=\operatorname{sgn}(\zeta \tau) \int_{\zeta \tau(x-r) \leq 0} e^{\zeta \tau(x-r))} \hat{G}_{j-1, \tau}(r, \zeta, t) d r .
$$

So

$$
\begin{aligned}
& \int_{-\infty}^{\infty} \hat{Q}_{j, \tau}(x, \zeta, t) d \zeta \\
& =\int_{-\infty}^{\infty} \operatorname{sgn}(\zeta \tau) \int_{\zeta \tau(x-r) \leq 0} e^{\zeta \tau(x-r))} \hat{G}_{j-1, \tau}(r, \zeta, t) d r d \zeta
\end{aligned}
$$

If $\tau \zeta>0$, then the above integral is equal to

$$
\int_{-\infty}^{\infty} \int_{-\infty}^{0} e^{\tau \zeta s} \hat{G}_{j-1, \tau}(x-s, \zeta, t) d s d \zeta
$$

For $\tau \zeta<0$, we get a similar but different formula. This explains the jump. So we obtain in a straightforward fashion the estimate independent of $\tau$ :

$$
\left\|\hat{Q}_{j, \tau}\right\|_{1} \leq\left\|\hat{G}_{j-1, \tau}\right\|_{2}=\left\|\hat{A}_{\tau} * \hat{Q}_{j-1, \tau}\right\|_{2} \leq\left\|\hat{A}_{\tau}\right\|_{2}\left\|\hat{Q}_{j-1, \tau}\right\|_{1} .
$$

The norms used are $\|\cdot\|_{1}$ as the $L_{x}^{\infty} L_{\zeta}^{1}$ and $\|\cdot\|_{2}$ as the $L_{(x, \zeta)}^{1}$ norm. Given these estimates, the solution

$$
\hat{Q}(x, \zeta, t)=\sum_{j \geq 0} \hat{Q}_{j}(x, \zeta, t)
$$

exists in $L_{x}^{\infty} L_{\zeta}^{1}$ when $\left\|\hat{A}_{\tau}\right\|_{2} \leq 1$. But it is easy to see that when the $W^{2,1}$ norm of $(A, \phi)$ is small, this inequality is satisfied. Furthermore, $\|\hat{Q}\|_{1}$ 
bounds $\|Q\|_{\infty}$ and existence follows. Iterating these same estimates in the various derivatives of $Q$ will give estimates on the higher norms, although we do not expect or require smallness in the higher derivatives and weights.

\section{References}

[1] C.K. Anand, Ward's solitons, Geom. Topol. 1 (1997), 9-20.

[2] C.K. Anand, Ward's solitons II, Exact solutions, Canad. J. Math. 50 (1998), 11191137.

[3] M.F. Atiyah and N.J. Hitchin, The geometry and dynamics of magnetic monopoles, Princeton University Press, Princeton, New Jersey, 1988.

[4] R. Beals and R.R. Coifman, Multidimensional inverse scattering and nonlinear partial differential equations, Proc. Symp. Pure Math. 43 (1985), 45-70.

[5] R. Beals and R.R. Coifman, Linear spectral problems, non-linear equations and the $\bar{\partial}$-method, Inverse Problems 5 (1989), 87-130.

[6] B. Dai and C.L. Terng, Bäcklund transformation, Ward solitons, and unitons, arXiv:math.DG/0405363.

[7] A.S. Fokas and T.A. Ioannidou, The inverse spectral theory for the Ward equation and for the $2+1$ chiral model, Comm. Appl. Anal. 5(2) (2001), 235-246.

[8] N.J. Hitchin, On the construction of monopoles, Comm. Math. Phys. 89 (1983), 145190.

[9] N.J. Hitchin, The self-duality equations on a Riemann surface, Proc. London Math. Soc. 55 (1987), 59-126.

[10] T. Ioannidou, Soliton solutions and nontrivial scattering in an integrable chiral model in $(2+1)$ dimensions, J. Math. Phys. 37 (1996), 3422-3441.

[11] T. Ioannidou and W. Zakrzewski, Solutions of the modified chiral model in $(2+1)$ dimensions, J. Math. Phys. 39(5) (1998), 2693-2701.

[12] T. Ioannidou and W. Zakrzewski, Lagrangian formulation of the general modified chiral model, Phys. Lett. A 249 (1998), 303-306.

[13] S.V. Manakov and V.E. Zakharov, Three-dimensional model of relativistic-invariant theory, integrable by the inverse scattering transform, Lett. Math. Phys. 5 (1981), 247-253.

[14] A. Pressley and G. Segal, Loop groups, Oxford University Press, 1986.

[15] T. Tao, Global regularity of wave maps. II. Small energy in two dimensions, Comm. Math. Phys. 224(2) (2001), 443-544.

[16] D. Tataru, The wave maps equation, Bull. Amer. Math. Soc. 41(2) (2004), 185-204.

[17] C.L. Terng and K. Uhlenbeck, Poisson actions and scattering theory for integrable systems. Surveys in differential geometry: integrable systems, 315-402, Surv. Diff. Geom., IV, International Press, Boston, MA, 1998.

[18] C.L. Terng and K. Uhlenbeck, Bäcklund transformations and loop group actions, Comm. Pure Appl. Math. 53 (2000), 1-75.

[19] K. Uhlenbeck, Harmonic maps into Lie groups (classical solutions of the chiral model), J. Differential Geom., 30 (1989), 1-50.

[20] K. Uhlenbeck, On the connection between harmonic maps and the self-dual Yang-Mills and the sine-Gordon equations, J. Geom. Phys. 8 (1992), 283-316.

[21] J. Villarroel, The inverse problem for Ward's system, Stud. Appl. Math. 83 (1990), 211-222.

[22] R.S. Ward, Soliton solutions in an integrable chiral model in $2+1$ dimensions, J. Math. Phys. 29 (1988), 386-389.

[23] R.S. Ward, Classical solutions of the chiral model, unitons, and holomorphic vector bundles, Comm. Math. Phys. 128 (1990), 319-332. 
[24] R.S. Ward, Nontrivial scattering of localized solutions in a $(2+1)$-dimensional integrable systems, Phys. Letter A 208 (1995), 203-208.

[25] R.S. Ward, Integrable systems and twistors, in 'Integrable Systems', Oxf. Grad. Texts Math., 4 (1999), 121-134.

[26] V.E. Zakharov and A.V. Mikhailov, A. Relativistically invariant two dimensional models of fields theory which are integrable by means of the inverse scattering problem method, Sov. Phys. JETP 47(6) (1978), 1017-1027.

LMam, School of Mathematical Sciences, Peking University,

BeiJing 100871, P.R. China

E-mail address: daibo@math.pku.edu.cn

Department of Mathematics, University of California at Irvine, IRvine, CA 92697-3875

E-mail address: cterng@math.uci.edu

Department of Mathematics, University of Texas at Austin, Austin, TX 78712

E-mail address: uhlen@math.utexas.edu 